\title{
L183 (L134N) revisited
}

\section{The gas depletion $\star, \star \star$}

\author{
L. Pagani ${ }^{1}$, J.-R. Pardo ${ }^{2}$, A. J. Apponi ${ }^{3}$, A. Bacmann ${ }^{4}$, and S. Cabrit ${ }^{1}$ \\ 1 LERMA \& UMR 8112 du CNRS, Observatoire de Paris, 61 Av. de l'Observatoire, 75014 Paris, France \\ e-mail: laurent.pagani@obspm.fr \\ 2 Instituto de Estructura de la Materia, Dpto de Física Molecular CSIC, Madrid, Spain \\ 3 University of Arizona, Steward Observatory, 933 N. Cherry Ave., Tucson, AZ 85721, USA \\ ${ }^{4}$ Observatoire de Bordeaux, BP 89, 33270 Floirac, France
}

Received 6 April 2004 / Accepted 27 July 2004

\begin{abstract}
We present a detailed study of the gas depletion in L183 (= L134N) for a set of important species, namely, CO, CS, $\mathrm{SO}, \mathrm{N}_{2} \mathrm{H}^{+}$and $\mathrm{NH}_{3}$. We show that all these species are depleted at some level. This level seems to depend mostly on a density threshold rather than on dust opacity. Therefore UV shielding would not be a main factor in the triggering of depletion. Our data suggest that $\mathrm{CO}, \mathrm{CS}$ and $\mathrm{SO}$ depletion happen at densities of $\sim 3 \times 10^{4} \mathrm{~cm}^{-3}$, while $\mathrm{N}_{2} \mathrm{H}^{+}$and $\mathrm{NH}_{3}$ seem to deplete at densities close to $10^{6} \mathrm{~cm}^{-3}$. The latter result is consistent with the Bergin \& Langer $\left(1997\right.$, ApJ, 486, 316) polar $\left(\mathrm{H}_{2} \mathrm{O}\right)$ ice case but not with the more recent models of Aikawa et al. (2003, ApJ, 593, 906). CS depletion occurs much below its (J:2-1) critical density, $\left(7 \times 10^{5} \mathrm{~cm}^{-3}\right)$ and therefore makes this species unsuitable to study the density structure of many dark cloud cores.
\end{abstract}

Key words. ISM: abundances - ISM: molecules - ISM: dust, extinction - ISM: individual: objects: L183 (L134N) radio lines: ISM

\section{Introduction}

In the very cold, shielded interior of dark molecular clouds, most molecules except $\mathrm{H}$-only species and possibly $\mathrm{N}$-carriers are expected to stick to grains and thus disappear from the gas phase. This has been anticipated as early as a few years after the discovery of $\mathrm{CO}$. Because $\mathrm{CO}$ was predicted to deplete onto grains at temperatures below $20 \mathrm{~K}$, it was not clear why it could still be seen in $10 \mathrm{~K}$ clouds. Yet, no clear trace of depletion could be found for any species until facilities for observing and measuring cold dust became available. In the mid-nineties, bolometer arrays (MAMBO at $1.2 \mathrm{~mm}$, SCUBA at 850 and $450 \mu \mathrm{m}$ ) have allowed astronomers to detect dense cold dust cores which had escaped all searches so far because $\mathrm{C}^{18} \mathrm{O}$, which was supposed to trace gas column-density in cold molecular clouds and thus locate cores via their high mass content, was in fact depleted inside these quiescent cores. Cores were thus found from dust emission eventually and more recently via near or mid infrared dust absorption. As a direct consequence, CO (and other molecules) depletion was eventually revealed. While depletion itself is no longer questioned,

* Based on observations made with the CFHT, the Iram 30-m and the ARO 12-m (formerly NRAO 12-m).

$\star \star$ Appendix A is only available in electronic form at http://www.edpsciences.org the physical conditions it requires for different species are not yet well-established. Polar molecules such as $\mathrm{H}_{2} \mathrm{O}$ freeze onto grains at temperatures below $\sim 100 \mathrm{~K}$ and apolar molecules (like CO) below $\sim 20 \mathrm{~K}$, but $\mathrm{N}$-bearing species, such as $\mathrm{NH}_{3}$ and $\mathrm{N}_{2} \mathrm{H}^{+}$do not seem to experience this as they are seen deep inside the cores and could even act as surrogate dust tracers. It is thus necessary to track depletion in as many cold clouds as possible to shed light on the details of the depletion mechanism.

A second reason to study depletion is linked to astrochemistry. Without taking depletion effects into account, all species are considered to be spatially coexistent when they are not $\left(\mathrm{N}_{2} \mathrm{H}^{+}\right.$cannot coexist with $\mathrm{CO}$ e.g., Bergin \& Langer 1997; Caselli 2002) and therefore relative abundances are not correctly evaluated. This is one of the reasons why previous abundance estimates made towards L183 by Swade (1989b) and by Dickens et al. (2000) should be considered very cautiously before checking astrochemical models against them. We have therefore undertaken to revisit this source in order to establish it as a true reference for chemical models.

Depletion was revealed in L183 for the first time by Pagani et al. (2002, hereafter Paper I) and independently confirmed by Juvela et al. (2002). However, in both works the depletion estimates were done by comparison with the ISOPHOT 100 and $200 \mu \mathrm{m}$ data which only probe a thin outer layer $\left(0-15 A_{\mathrm{V}}\right)$ of cold $(12-17 \mathrm{~K})$ dust. Therefore the depletion along the 
line-of-sight was evaluated to a factor of 1.5-2 only. As we showed in a subsequent paper (Pagani et al. 2004, hereafter Paper II), these early estimates are not correct because they ignore the bulk of the dust, which is at $T<10 \mathrm{~K}$ and does not emit at $200 \mu \mathrm{m}$. Therefore, depletion has been strongly underevaluated in these earlier works. We have now obtained (Paper II) a composite Mid- and Near-Infrared dust absorption map which traces all the dust column density, together with a map of the dust emission at $1.2 \mathrm{~mm}$, and therefore we can work out more precisely the depletion effects in this source.

In this paper, we will present in Sect. 2 the molecular line observations we have made, analyze them in Sect. 3 and discuss the depletion effects we find by comparison with our dust map from Paper II in Sect. 4. In Sect. 5, we will draw our conclusions.

\section{Observations}

We have observed four species, namely $\mathrm{CO}, \mathrm{CS}, \mathrm{SO}$ and $\mathrm{N}_{2} \mathrm{H}^{+}$, and for each species at least two isotopomers and for each isotopomer at least 2 transitions (except for the $\mathrm{N}_{2} \mathrm{H}^{+}$isotopomer for which we have only a few $J: 3-2$ observations with low signal-to-noise ratio). For the goals of the present work, we concentrate on a small subset of these observations (Table 1) complemented with observations from Tiné et al. (2000). The remaining observations will be published in subsequent papers. The reference position of the source in this study is: $15^{\mathrm{h}} 54^{\mathrm{m}} 06.6^{\mathrm{s}},-2^{\circ} 52^{\prime} 19^{\prime \prime}(\mathrm{J} 2000)$ which is the L134N reference position given by e.g. Swade (1989a) and the LSR velocity is $+2.5 \mathrm{~km} \mathrm{~s}^{-1}$. Note however that the SIMBAD position for this source is $15^{\mathrm{h}} 54^{\mathrm{m}} 09.2^{\mathrm{s}},-2^{\circ} 51^{\prime} 39^{\prime \prime}(\mathrm{J} 2000)$ and that a few other reference positions are also found in the literature. We would suggest using the central position of the dust peak from now on as the reference in further work: $15^{\mathrm{h}} 54^{\mathrm{m}} 08.5^{\mathrm{s}}$, $-2^{\circ} 52^{\prime} 43^{\prime \prime}(\mathrm{J} 2000)$.

The subset of observations presented here have been carried out with either the Kitt Peak 12-m (formerly owned by NRAO, now operated by the University of Arizona, hereafter KP 12-m) or the IRAM 30-m.

\subsection{Kitt Peak 12-m data}

Observations started in December 1999 at Kitt Peak and spanned a seven month period. Complementary observations were obtained in January 2001. The Kitt Peak 12-m is equipped with two-polarization receivers at 1.3, 2 and $3 \mathrm{~mm}$ with filter banks and a versatile autocorrelator in parallel as backends. We used $100 \mathrm{kHz}$ filters all the time and the 32K-channel autocorrelator. Pointing (and focus) were checked on planets when available or 3C 273 and 3C 279 and found to be stable within $5^{\prime \prime}$. Pointing was checked once for short observing periods and twice for long periods (up to $7 \mathrm{~h}$ ). Except for $\mathrm{N}_{2} \mathrm{H}^{+}$, all observations were conducted in frequency switch mode. The $\mathrm{N}_{2} \mathrm{H}^{+}(J: 1-0)$ line spans almost $20 \mathrm{~km} \mathrm{~s}^{-1}$ and is thus better observed in position switch mode. The off position was taken $10^{\prime}$ northwest of the source where no $\mathrm{C}^{18} \mathrm{O}$ emission is seen. The reference position was observed every $30 \mathrm{~min}$ or so to check for gain variations. For the weak $\mathrm{C}^{17} \mathrm{O}$ line, we made
Table 1. Observational parameters and sensitivity.

\begin{tabular}{lcccc}
\hline \hline Molecule & Transition & $\begin{array}{c}\text { Frequency } \\
(\mathrm{MHz})\end{array}$ & $\begin{array}{c}\text { Beam size } \\
(\operatorname{arcsec})\end{array}$ & $\begin{array}{c}\mathrm{rms}^{a} \\
(\mathrm{~K})\end{array}$ \\
\hline $\mathrm{C}^{18} \mathrm{O}$ & $J: 1-0$ & 109782.163 & $57_{\mathrm{KP}}$ & 0.11 \\
$\mathrm{C}^{18} \mathrm{O}^{b}$ & $J: 1-0$ & 109782.163 & $23_{\mathrm{I}}$ & 0.059 \\
$\mathrm{C}^{18} \mathrm{O}$ & $J: 2-1$ & 219560.327 & $28_{\mathrm{KP}}$ & $0.26^{e}$ \\
$\mathrm{C}^{18} \mathrm{O}$ & $J: 2-1$ & 219560.327 & $12_{\mathrm{I}}$ & $0.12^{e}$ \\
$\mathrm{C}^{17} \mathrm{O}$ & $J: 1-0$ & 112359.277 & $56_{\mathrm{KP}}$ & 0.034 \\
$\mathrm{C}^{32} \mathrm{~S}$ & $J: 2-1$ & $97980.950^{c}$ & $64_{\mathrm{KP}}$ & 0.088 \\
${ }^{32} \mathrm{SO}$ & $J_{N}: 3_{2}-2_{1}$ & 99299.879 & $63_{\mathrm{KP}}$ & 0.12 \\
$\mathrm{~N}_{2} \mathrm{H}^{+}$ & $J: 1-0$ & $93173.384^{d}$ & $67_{\mathrm{KP}}$ & 0.14 \\
$\mathrm{~N}_{2} \mathrm{H}^{+}$ & $J: 1-0$ & $93173.384^{d}$ & $27_{\mathrm{I}}$ & 0.037 \\
$\mathrm{~N}_{2} \mathrm{D}^{+}$ & $J: 2-1$ & $154217.096^{d}$ & $16_{\mathrm{I}}$ & 0.050 \\
$\mathrm{~N}_{2} \mathrm{D}^{+}$ & $J: 3-2$ & $231321.757^{d}$ & $12_{\mathrm{I}}$ & 0.097 \\
$\mathrm{NH}_{2} \mathrm{D}_{\text {para }}{ }^{b}$ & $J_{K K^{\prime}}: 1_{11}-1_{01}$ & $110153.587^{d}$ & $23_{\mathrm{I}}$ & 0.054 \\
\hline
\end{tabular}

${ }^{a}$ Median value over the map.

${ }^{b}$ Data partly from Tiné et al. (2000).

${ }^{c}$ Frequency is taken from Pagani et al. (2001).

${ }^{d}$ Hyperfine components.

${ }^{e}$ Small map towards Peak E only.

I IRAM 30-m telescope.

KP NRAO/ARO KP 12-m.

long integrations, and gain was checked only once per session. Gain variations towards the reference position would have been undetectable for such low SNR spectra. Autocorrelator sampling was set to $6 \mathrm{kHz}$ (12 kHz resolution) which represents 30 to $36 \mathrm{~m} \mathrm{~s}^{-1}$ velocity resolution depending on the frequency. Data were Hanning smoothed afterwards to velocity resolution up to 0.1 or $0.2 \mathrm{~km} \mathrm{~s}^{-1}$ depending on the SNR needed. Whenever possible, the flux scale was checked on NGC 2264 (for SO lines, e.g.) or IRC+10216 at the beginning of the observations. Mapping was done with 1' steps except in some critical places (such as the dust core) where $30^{\prime \prime}$ step sampling was preferred. We mapped most of the lines on an area $15^{\prime} \times 15^{\prime}$, large enough to cover the entire L183 core (but the cloud connects with L134 southward). In a few cases, we stopped the mapping before, when the line became too weak $\left(\mathrm{N}_{2} \mathrm{H}^{+}\right.$in most directions and ${ }^{32} \mathrm{SO}$ northward). The single sideband system temperature $\left(T_{\text {sys }}(\mathrm{SSB})\right)$ was in the range $100-200 \mathrm{~K}$.

\subsection{IRAM 30-m data}

IRAM 30-m data were collected in April, May, December 2003 and May 2004. The IRAM 30-m is equipped with two polarization receivers at 1.1, 1.3, 2 and $3 \mathrm{~mm}$. It has filter banks and a versatile autocorrelator. The system allows to observe up to 4 different lines simultaneously. We worked with a frequency resolution of $20 \mathrm{kHz}$ at 2 and $3 \mathrm{~mm}$, giving us a velocity resolution around $50 \mathrm{~m} \mathrm{~s}^{-1}$ at $3 \mathrm{~mm}$ and $35 \mathrm{~m} \mathrm{~s}^{-1}$ at $2 \mathrm{~mm}$. At $1.3 \mathrm{~mm}$, we worked with $40 \mathrm{kHz}$ resolution to keep the same $50 \mathrm{~m} \mathrm{~s}^{-1}$ velocity resolution. Pointing and focus were checked every $90 \mathrm{~min}$ on close-by continuum sources. The observations have been performed in frequency switch mode. We observed a few positions in $\mathrm{N}_{2} \mathrm{H}^{+}$and $\mathrm{N}_{2} \mathrm{D}^{+}$in April and May 2003 . 

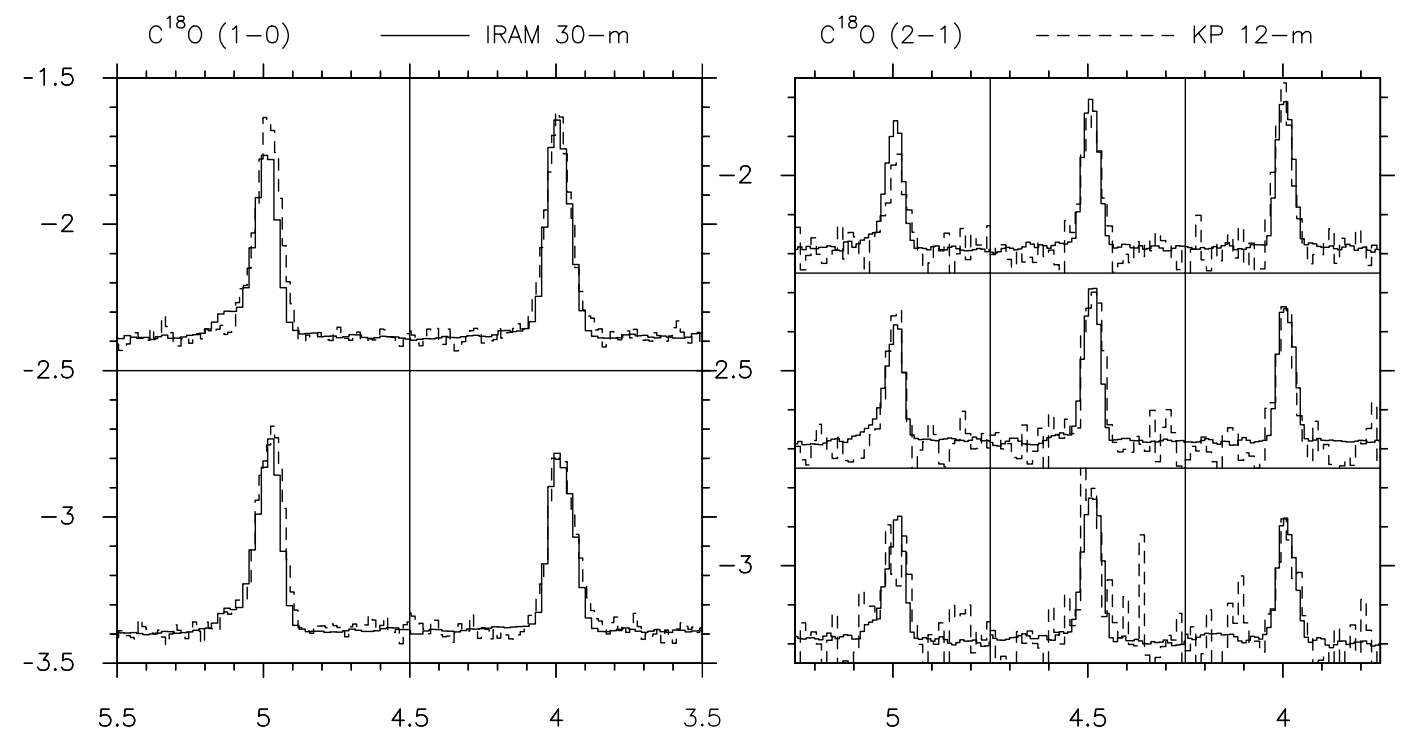

Fig. 1. Comparison of $\mathrm{C}^{18} \mathrm{O}(J: 1-0)(l e f t)$ and $(J: 2-1)$ (right) spectra (towards peak E) from the Kitt Peak (KP) 12-m and IRAM 30-m telescopes after smoothing the latter to the KP 12-m resolution ( $1^{\prime}$ for the $(J: 1-0)$ and $30^{\prime \prime}$ for the $(J: 2-1)$ transitions). Coordinates are offsets in arcmin from reference position. $X$ axis (velocity) scale is from 0 to $5 \mathrm{~km} \mathrm{~s}^{-1}$, and $Y$ axis $\left(T_{r}^{*}\right)$ scale is from -0.5 to $4.0 \mathrm{~K}(J: 1-0)$ and from -0.3 to $2.2 \mathrm{~K}$ $(J: 2-1)$.

$T_{\text {sys }}(\mathrm{SSB})$ were $100 \mathrm{~K}$ at $3 \mathrm{~mm}, 300 \mathrm{~K}$ at $2 \mathrm{~mm}$ and 400 to $700 \mathrm{~K}$ at $1.3 \mathrm{~mm}$. The reader is referred to Tiné et al. (2000) for the description of their observations. We performed further observations of the $\mathrm{N}_{2} \mathrm{D}^{+}(\mathrm{J}: 2-1)$ line in December 2003 to improve and enlarge Tiné's map to a size of $100^{\prime \prime} \times 200^{\prime \prime}$. We also mapped the $\mathrm{N}_{2} \mathrm{D}^{+}(J: 3-2)$ transition. For both transitions, $T_{\text {sys }}$ were similar to what we obtained in Spring 2003. The $1.3 \mathrm{~mm}$ receiver we used was HERA (9 pixels spaced by $\left.24^{\prime \prime}\right)$ with the new autocorrelator, VESPA. We observed the $\mathrm{N}_{2} \mathrm{D}^{+}(\mathrm{J}: 2-1)$ line with $10 \mathrm{kHz}$ resolution $\left(=20 \mathrm{~m} \mathrm{~s}^{-1}\right)$ and the $\mathrm{N}_{2} \mathrm{D}^{+}(\mathrm{J}: 3-2)$ line with $40 \mathrm{kHz}$ resolution $\left(=50 \mathrm{~m} \mathrm{~s}^{-1}\right)$.

\subsection{Calibration}

The KP 12-m telescope forward scattering and spillover efficiency $\left(\eta_{\text {fss }}\right.$, Kutner \& Ulrich 1981) has been measured to be 0.64 at all wavelengths. This efficiency allows to calibrate our data in the $T_{r}^{*}$ scale if we suppose the beam coupling to the source to be 1 . Though the exact definition of the $T_{r}^{*}$ scale depends on what angular size we use to measure $\eta_{\text {fss }}$, its general acceptation (or its closest approximation, $\eta_{\text {moon }}$ ) applies to calibrate signals from extended sources larger than the main beam, such as L183 (see Teyssier et al. 2002, for a recent discussion on this point). For the IRAM $30-\mathrm{m}$, the forward efficiency $\left(\eta_{\mathrm{f}}\right)$ is identical to $\eta_{\text {moon }}$ which means that for extended sources such as L183, it is less erroneous to use $\eta_{\mathrm{f}}$ than to use the main beam efficiency, $\eta_{\mathrm{MB}}$, which introduces too large a correction. In other words, for the IRAM $30-\mathrm{m}, T_{a}^{*} \approx T_{r}^{*}$.

Indeed, we find (Fig. 1) that for $\mathrm{C}^{18} \mathrm{O}(J: 1-0)$ and $(J: 2-1)$, the IRAM 30-m telescope gives comparable results to the KP 12-m telescope after convolution at the KP 12-m resolution if we use the IRAM $30-\mathrm{m} T_{a}^{*}$ scale and the KP $12-\mathrm{m}$ $T_{r}^{*}$ scale. This indicates that the calibration of both telescopes is consistent for extended emission tracers. If we had applied the $\eta_{\mathrm{MB}}$ correction for both telescopes then the

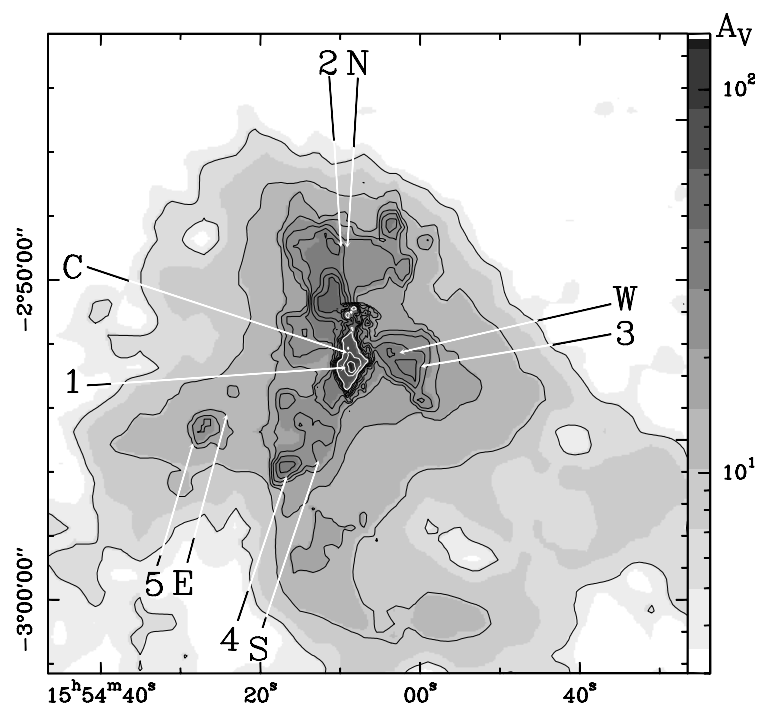

Fig. 2. Dust map from Paper II (in log grey scale). Coordinates are J2000 equatorial. Letters C, E, N, S, and W mark the molecular peaks while numbers 1 to 5 mark the corresponding dust peaks (see text). Dust peak \#2 is not visible in this map but only in the dust emission map at $1.2 \mathrm{~mm}$ (see Paper II). Contours are from 5 to 50 by steps of 5 mag (black contours) and 60 to 120 by steps of $30 \mathrm{mag}$ (white contours).

IRAM 30-m (J:1-0) line would have been $23 \%$ stronger than the KP 12-m line while the KP 12-m (J:2-1) line would have been $26 \%$ stronger than the IRAM $30-\mathrm{m}$ line, which would have lead to considerable discrepancies in the $(J: 2-1) /(J: 1-0)$ line ratios.

\section{Results}

Because of its importance in this work, we redisplay first the dust map (Fig. 2) we obtained in Paper II with arrows marking 


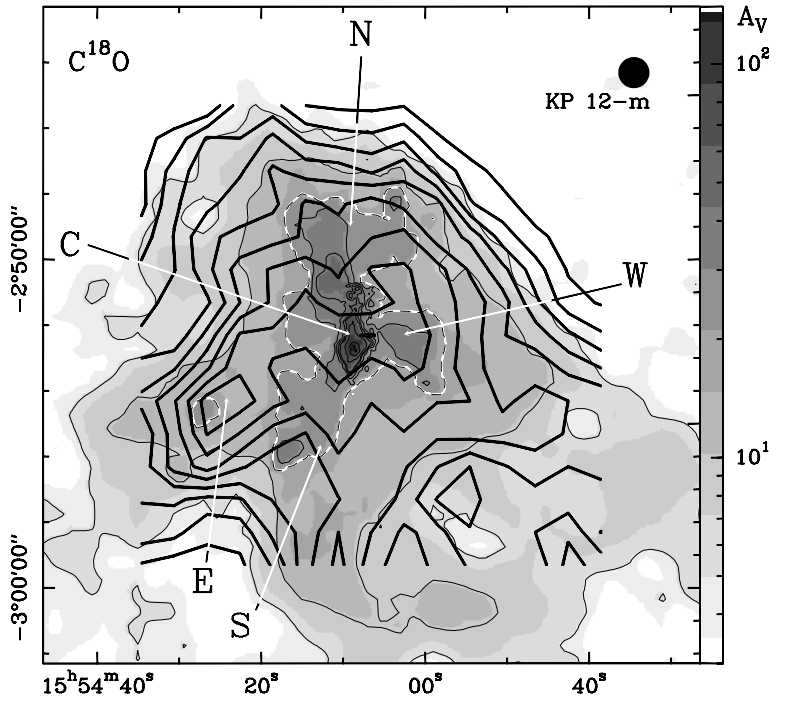

Fig. 3. $\mathrm{C}^{18} \mathrm{O}(\mathrm{J}: 1-0)$ integrated intensity contours (from the KP 12-m telescope) superimposed on the dust map from Paper II (in log grey scale). Coordinates are $\mathrm{J} 2000$ equatorial. $\mathrm{C}^{18} \mathrm{O}$ levels: 0.2 to 1.8 by $0.2 \mathrm{~K} \mathrm{~km} \mathrm{~s}^{-1}\left(\mathrm{rms} \approx 35 \mathrm{mK} \mathrm{km} \mathrm{s}^{-1}\right)$. The white and black dashed contour represents the $A_{\mathrm{V}}=20$ mag level. Letters C, E, N, S, and W mark the molecular peaks (see text) and the KP 12-m beam size is displayed in the top right corner.

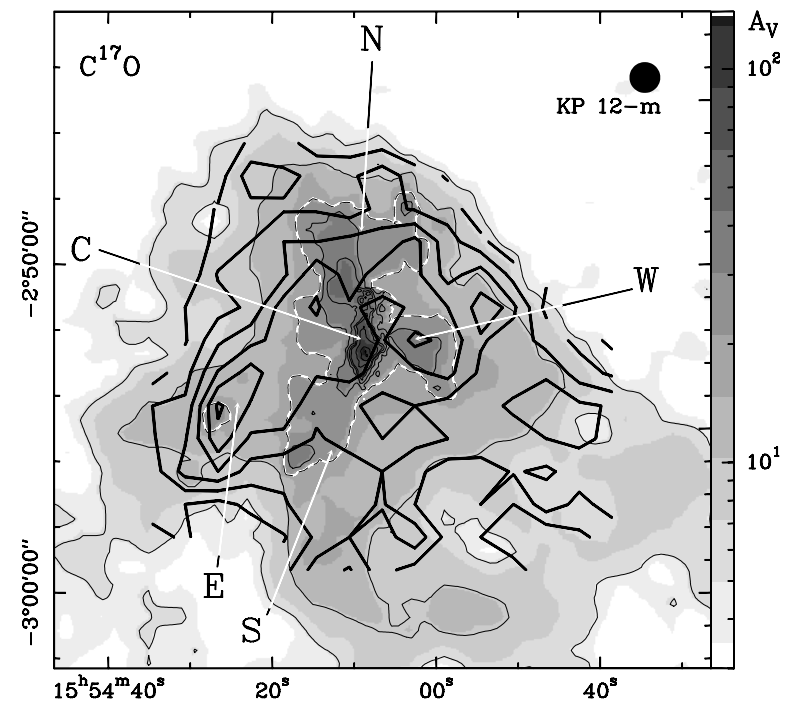

Fig. 4. Same as Fig. 3 for $\mathrm{C}^{17} \mathrm{O}(J: 1-0)$. $\mathrm{C}^{17} \mathrm{O}$ levels: 0.1 to 0.7 by $0.1 \mathrm{~K} \mathrm{~km} \mathrm{~s}^{-1}\left(\mathrm{rms} \approx 30 \mathrm{mK} \mathrm{km} \mathrm{s}{ }^{-1}\right)$.

both the places of the molecular peaks (C, N, S and W) described by Dickens et al. (2000) to which we have added a fifth molecular peak, E (see below), and the places of the nearby dust peaks which we have numbered from 1 to 5 .

\subsection{Large-scale Kitt Peak maps}

Figures 3-7 show $\mathrm{C}^{18} \mathrm{O}, \mathrm{C}^{17} \mathrm{O}, \mathrm{C}^{32} \mathrm{~S},{ }^{32} \mathrm{SO}$, and $\mathrm{N}_{2} \mathrm{H}^{+}$emission intensity superposed as contours on the dust map image from Paper II (see Fig. 2). For the dust map, the $A_{\mathrm{V}}=20$ mag contour is emphasized.

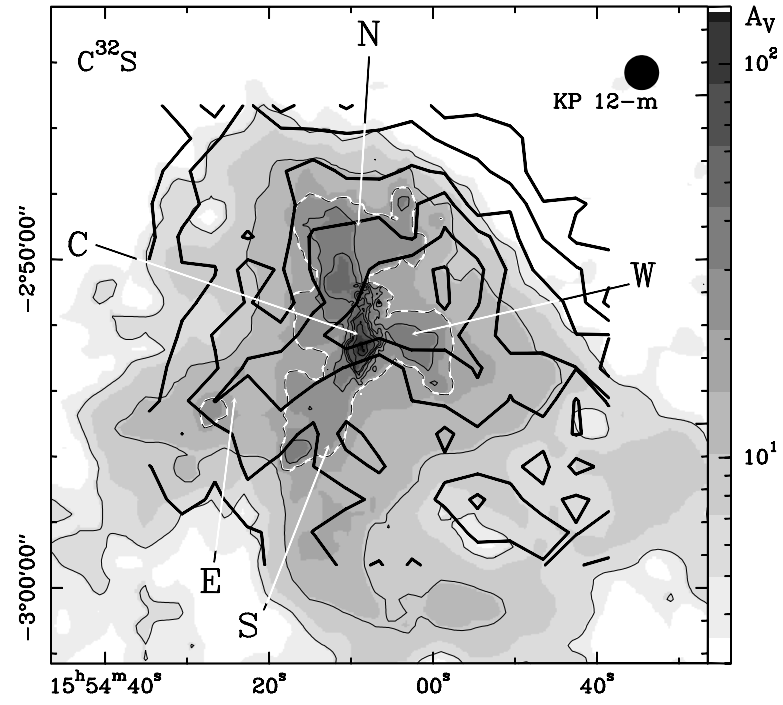

Fig. 5. Same as Fig. 3 for $C^{32} S(J: 2-1) . C^{32} S$ levels: 0.1 to 1.1 by $0.2 \mathrm{~K} \mathrm{~km} \mathrm{~s}^{-1}\left(\mathrm{rms} \approx 45 \mathrm{mK} \mathrm{km} \mathrm{s}^{-1}\right)$.

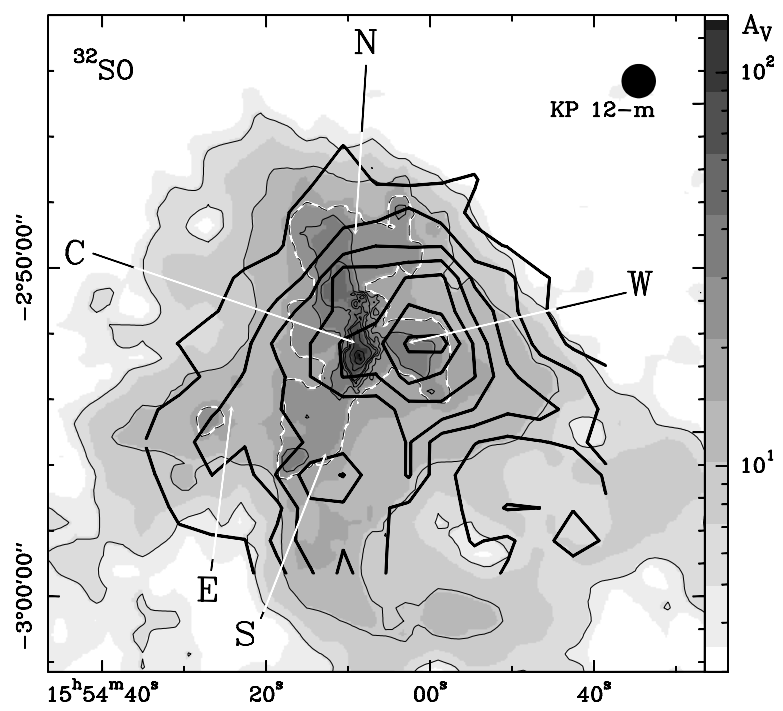

Fig. 6. Same as Fig. 3 for ${ }^{32} \mathrm{SO}\left(J_{N}: 3_{2}-2_{1}\right) .{ }^{32} \mathrm{SO}$ levels: 0.3 to 2.1 by $0.3 \mathrm{~K} \mathrm{~km} \mathrm{~s}^{-1}\left(\mathrm{rms} \approx 60 \mathrm{mK} \mathrm{km} \mathrm{s}{ }^{-1}\right)$.

For both $\mathrm{C}^{18} \mathrm{O}$ (Fig. 3) and $\mathrm{C}^{17} \mathrm{O}$ (Fig. 4) (J:1-0) lines, it is clear that none of the four molecular peaks $(\mathrm{N}, \mathrm{S}, \mathrm{C}, \mathrm{W})$ described by Dickens et al. (2000) is visible (except a very small $\mathrm{C}^{18} \mathrm{O}$ peak towards Peak $\mathrm{C}$ but with no counterpart in $\mathrm{C}^{17} \mathrm{O}$ ). To the east, there is a $\mathrm{C}^{18} \mathrm{O} / \mathrm{C}^{17} \mathrm{O}$ peak which coincides with our dust peak \# 5 (Fig. 2 and Paper II) and which we label E to keep consistency with Dickens et al. (2000, this peak is outside their $\mathrm{C}^{18} \mathrm{O}$ map). Interestingly enough, this dust peak \# 5 reaches an optical extinction of $A_{\mathrm{V}} \approx 25 \mathrm{mag}$. While $\mathrm{C}^{18} \mathrm{O}$ does show opacity effects (as mentioned in Paper I), it is not the case for $\mathrm{C}^{17} \mathrm{O}$ (the main hyperfine component is approximately ten times weaker that the $\mathrm{C}^{18} \mathrm{O}$ line). Both isotopomers show the same features and thus the absence of the four N, S, $\mathrm{C}$ and $\mathrm{W}$ peaks is not due to opacity effects but is real.

The $C^{32} S(J: 2-1)$ line emission map (Fig. 5) shows no correlation with any of the five peaks but only a general increase towards the center of the cloud. However, the emission 


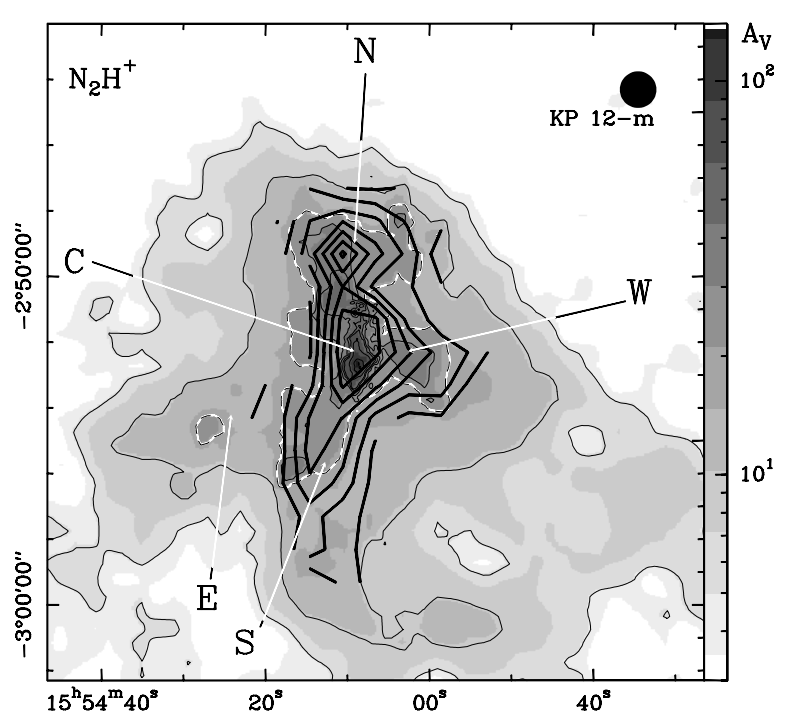

Fig. 7. Same as Fig. 3 for $\mathrm{N}_{2} \mathrm{H}^{+}(J: 1-0) . \mathrm{N}_{2} \mathrm{H}^{+}$levels: 0.4 to 2.8 by $0.4 \mathrm{~K} \mathrm{~km} \mathrm{~s}^{-1}\left(\mathrm{rms} \approx 75 \mathrm{mK} \mathrm{km} \mathrm{s}^{-1}\right)$.

peak is situated north-west of peak W, noticeably outside of the dust cloud core and the line is obviously optically thick as its self-reversed profile indicates ${ }^{1}$ and the question of peak hiding in the map arises again.

The ${ }^{32} \mathrm{SO}\left(J_{N}: 3_{2}-2_{1}\right)$ line emission map (Fig. 6) shows a good correlation with two of the peaks (W and $\mathrm{S}$ ) with a small extension towards peak $\mathrm{C}$. It does not extend as far to the north as the $\mathrm{CO}$ and $\mathrm{CS}$ lines but is otherwise as extended as $\mathrm{C}^{18} \mathrm{O}$. However, the strongest SO peak is the $\mathrm{W}$ peak and not the $\mathrm{C}$ peak while the dust content is 4 times as high towards dust peak 1 than towards dust peak 3 (see Fig. 2).

Finally, the $\mathrm{N}_{2} \mathrm{H}^{+}(J: 1-0)$ map (Fig. 7) is clearly different from the previous four as its extent is much more limited. It is rather well correlated with the dust content above $A_{\mathrm{V}}=20$ mag with two prominent peaks coincident with molecular peaks $\mathrm{N}$ and $\mathrm{C}$ and two extensions reaching peaks $\mathrm{W}$ and $\mathrm{S}$. Peak $\mathrm{E}$ is conspicuously absent (a very weak line, $0.15 \mathrm{~K}$ peak intensity a $4 \sigma$ detection - has been detected though). It is remarkable then that the only $\mathrm{C}^{18} \mathrm{O} / \mathrm{C}^{17} \mathrm{O}$ peak coinciding with a dust peak is the only peak which hardly emits in $\mathrm{N}_{2} \mathrm{H}^{+}(J: 1-0)$. Overall, $\mathrm{CO}$ and $\mathrm{N}_{2} \mathrm{H}^{+}$maps are completely unrelated.

While all these differing features could have looked puzzling fifteen years ago when looking at Swade's maps (Swade 1989a), we now know that the main reason behind these differences is the depletion of CO, CS and SO onto grains, as we will discuss below. Tafalla et al. (2002) recently reached the same conclusions towards other pre-stellar cores.

\subsection{Small scale maps and cut}

These maps concern data taken with the IRAM 30-m telescope with resolutions in the range 11-23".

Tiné et al. (2000) mapped a small region around the central molecular peak (peak C) with the IRAM 30-m

\footnotetext{
${ }^{1}$ Dickens et al. (2000) advocated the existence of two independent peaks instead of a self-absorption feature, due to the use of an erroneous CS frequency, see Pagani et al. (2001).
}

which we redisplay in Fig. 8 along with the main core dust peak (Pagani et al. 2003; Paper II). The molecular data corresponds to $\mathrm{C}^{18} \mathrm{O}(J: 1-0), \mathrm{N}_{2} \mathrm{D}^{+}(J: 2-1)$ and $(J: 3-2)$ and para- $\mathrm{NH}_{2} \mathrm{D}\left(J_{K K^{\prime}}: 1_{11}-1_{01}\right)$ integrated line emission. The $\mathrm{N}_{2} \mathrm{D}^{+}(J: 2-1)$ map differs from the Tiné et al. (2000) original one because we display here our own observations instead of theirs. We also mapped the $\mathrm{N}_{2} \mathrm{D}^{+}(J: 3-2)$ line in December 2003 (a line which they failed to detect). $\mathrm{C}^{18} \mathrm{O}$ shows almost no variation and no correlation with the dust peak while the two deuterated species peak approximately at the central molecular peak (peak C) ${ }^{2}$, with only a weak extension towards the dust peak.

To better reveal the core depletion, we have also plotted these N-bearing species integrated intensities in a north-south cut across the dust peak (Fig. 9). We have also included some $\mathrm{N}_{2} \mathrm{H}^{+}(J: 1-0)$ data from the KP 12-m map and from sparse IRAM 30-m observations, not numerous enough to make a map. The intensity axis is arbitrary and is set to have approximately all species integrated intensities proportional to the dust emission on both sides of the dust peak. The dust peak clearly emerges outside the molecular peak.

\section{Depletion effects}

Two difficulties arise in the search for depletion effects. The first one is to have a correct estimate of the dust content. For example, Willacy et al. (1998) have used ISOPHOT 100 and $200 \mu \mathrm{m}$ data to estimate the dust content in the very cold source L1498. While the $\mathrm{C}^{18} \mathrm{O}$ depletion is unquestionable in that source, the dust is probably largely underestimated (as noted by the authors themselves) because the $200 \mu \mathrm{m}$ emission does not trace dust below $10 \mathrm{~K}$ as we also show in Paper II. As mentioned above, we (Paper I) and Juvela et al. (2002) met the same problem. The second difficulty is to have a correct estimate of the species column density. For example, in cold clouds, with high gas column densities, $\mathrm{C}^{18} \mathrm{O}$ can become optically thick. In fact, if it were not for depletion effects, optically thick $\mathrm{C}^{18} \mathrm{O}$ would be very wide-spread in dark clouds with optical extinction above $A_{\mathrm{V}} \approx 20 \mathrm{mag}$. One solution is to use $\mathrm{C}^{17} \mathrm{O}$ instead but the line is weak and difficult to map on large areas. Another solution is to map the $\mathrm{C}^{18} \mathrm{O}(\mathrm{J}: 2-1)$ and $(J: 3-2)$ transitions and run detailed radiative transfer models. It is a time-consuming task too and the result is not straightforward as it implies some interpretation of the data prior to diagnosing depletion effects. The question thus often arises to know whether the $\mathrm{C}^{18} \mathrm{O}$ intensity flattening commonly observed towards the cores of cold dark clouds is really due to depletion or instead to radiative transfer effects such as optically thick gas or extremely cold (5-6 K) gas which could induce the same behaviour. Tafalla et al. (2002) have shown that depletion is the only possible explanation for the five starless cores they have studied and most published cases usually show equivalent $\mathrm{C}^{17} \mathrm{O}$ behavior which rules out opacity effects. Low temperatures are usually thought difficult to justify from $\mathrm{NH}_{3}$ and $\mathrm{CO}$ measurements. Here we are going to discuss depletion effects for $\mathrm{CO}, \mathrm{CS}$ and $\mathrm{SO}$ on the

\footnotetext{
2 The low resolution of Dickens et al. (2000) data probably explain that their peak seems slightly displaced eastward.
} 


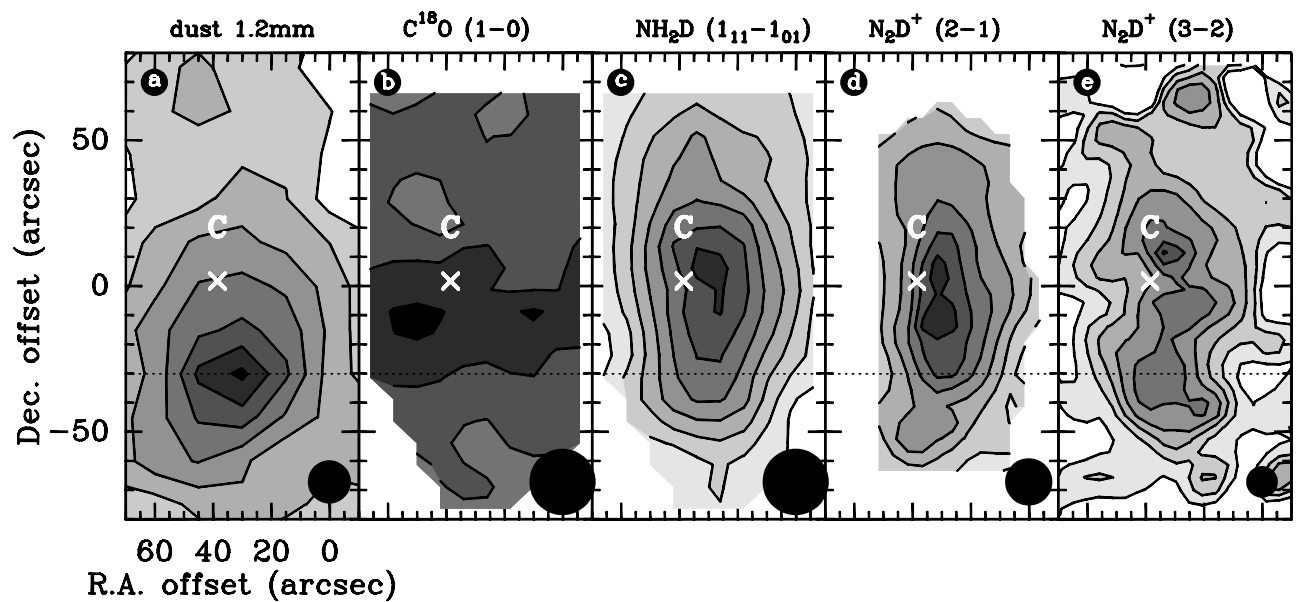

Fig. 8. a)-e). IRAM 30-m maps of the molecular peak C and dust peak 1 (see Paper II; Dickens et al. 2000). a) Dust peak emission measured at $1.2 \mathrm{~mm}$. Levels are 9 to 24 by $3 \mathrm{MJy} / \mathrm{Sr}$. b) $\mathrm{C}^{18} \mathrm{O}(J: 1-0)$ integrated intensity. Levels are 1.2 to 1.8 by $0.2 \mathrm{~K} \mathrm{~km} \mathrm{~s}^{-1}$. c) $\mathrm{NH}_{2} \mathrm{D}\left(J_{K K^{\prime}}: 1_{11}-1_{01}\right)$ integrated intensity. Levels are 0.15 to 1.05 by $0.15 \mathrm{~K} \mathrm{~km} \mathrm{~s}^{-1}$. d) $\mathrm{N}_{2} \mathrm{D}^{+}(\mathrm{J}: 2-1)$ integrated intensity. Levels are 0.2 to 1.4 by $0.2 \mathrm{~K} \mathrm{~km} \mathrm{~s} \mathrm{k}^{-1}$. e) $\mathrm{N}_{2} \mathrm{D}^{+}(\mathrm{J}: 3-2)$ integrated intensity. Levels are 0.05 to 0.35 by $0.05 \mathrm{~K} \mathrm{~km} \mathrm{~s}^{-1}$. The horizontal dashed line indicates where the dust peak should appear in declination for the maps of the different species and the cross marks the Dickens et al. (2000) molecular peak C. The black spots in the bottom right corners indicate the different map resolutions of the IRAM 30-m (from 11" to $23^{\prime \prime}$ ). Rms is $\approx 24 \mathrm{mK} \mathrm{km} \mathrm{s}^{-1}$ for maps b) and c), $40 \mathrm{mK} \mathrm{km} \mathrm{s}^{-1}$ for map d) and $28 \mathrm{mK} \mathrm{km} \mathrm{s}^{-1}$ for map e).

large scale and then the depletion effects we see with nitrogenbearing species $\left(\mathrm{N}_{2} \mathrm{H}^{+}, \mathrm{N}_{2} \mathrm{D}^{+}\right.$, and $\left.\mathrm{NH}_{2} \mathrm{D}\right)$.

\subsection{CO depletion}

If we plot the pixel-to-pixel comparison of the $\mathrm{C}^{18} \mathrm{O}$ integrated intensity versus the dust optical extinction and combine the two sets of $\mathrm{C}^{18} \mathrm{O}$ data (KP 12-m and IRAM 30-m), we obtain the very clear-cut picture of depletion of Fig. 10. The $A_{\mathrm{V}}$ data (Paper II) have been smoothed to each telescope resolution and thus the peak opacity is only $\sim 100$ mag now for a $23^{\prime \prime}$ beam and $\sim 50$ mag for a $60^{\prime \prime}$ beam. The turn-over is abrupt and occurs at $A_{\mathrm{V}} \approx 20 \mathrm{mag}$. This is a factor of 2-3 higher than the value found by Bergin et al. (2002) in B68 where $\mathrm{C}^{18} \mathrm{O}$ saturation starts at $A_{\mathrm{V}} \approx 7 \mathrm{mag}$ and becomes flat at $A_{\mathrm{V}} \approx 10 \mathrm{mag}$, or by Kramer et al. (1999) in IC 5146 and by Alves et al. (1999) in $\mathrm{L} 977$ where depletion is found to start at $A_{\mathrm{V}} \approx 10 \mathrm{mag}$ for both sources. Thus except for peak $\mathrm{E}$ (or dust peak 5) all the region inside the $A_{\mathrm{V}}=20$ mag contour is depleted of $\mathrm{CO}$ isotopomers. The $\mathrm{C}^{18} \mathrm{O}$ depletion surface covers $\sim 25^{\prime 2}$, a wide area indeed which explains the poor resemblance between the $\mathrm{C}^{18} \mathrm{O}$ map and maps of several other species in this source. A possible marginal increase of the $\mathrm{C}^{18} \mathrm{O}$ integrated intensity is still visible beyond the depletion turnover. A linear fit is not physical because the depletion probably increases going deeper into the core but the slope gives a good lower limit to the depletion we observe here. We have fitted the Kitt Peak data for $A_{\mathrm{V}}<$ 20 mag on the one hand and the whole IRAM data on the other hand (Fig. 10). We find a slope of 87 and $2.0 \mathrm{mK} \mathrm{km} \mathrm{s}^{-1} \mathrm{mag}^{-1}$ respectively. The decline in abundance from the region below to the region above $A_{\mathrm{V}} \approx 20$ mag is thus larger than $\sim 43$ on average (and the line of sight depletion is about 5 for the peak opacity). Below $A_{\mathrm{V}} \approx 20 \mathrm{mag}$, the $\mathrm{C}^{18} \mathrm{O}$ abundance is $\sim 1.1 \times$ $10^{-7}$, though the detailed Monte-Carlo analysis performed for two positions (Paper I) and which yields higher column densities than the simpler $\mathrm{LVG}$ analysis indicates a $\mathrm{C}^{18} \mathrm{O}$ abundance close to $1.4 \times 10^{-7}$, quite comparable to the value given by Frerking et al. (1982).

As discussed in Sect. 3, the comparison between $\mathrm{C}^{18} \mathrm{O}$ and $\mathrm{C}^{17} \mathrm{O}$ maps shows identical features between the two isotopomers and thus the opacity of the $\mathrm{C}^{18} \mathrm{O}(\mathrm{J}: 1-0)$ is relatively small and cannot explain the absence of molecular peaks towards the main dust peak. Low temperatures are not a better explanation since, on the one hand, $40^{\prime \prime}$ away from the dust peak, i.e. towards the $\mathrm{L} 134 \mathrm{~N}$ reference position, we have estimated the $\mathrm{C}^{18} \mathrm{O}$ total column density to be $2.7 \times 10^{15} \mathrm{~cm}^{-2}$ (Paper I), and, on the other hand, the line intensities of the $\mathrm{C}^{18} \mathrm{O}$ transitions change very little between the two positions, and therefore the $\mathrm{C}^{18} \mathrm{O}$ column density should be approximately the same towards the dust peak. From the dust peak estimated $A_{\mathrm{V}}$ $\left(\sim 100\right.$ mag for $23^{\prime \prime}$ resolution) and using $X\left(\mathrm{C}^{18} \mathrm{O}\right)=1.4 \times$ $10^{-7}$, we would have expected a total $\mathrm{C}^{18} \mathrm{O}$ column density of $\sim 1.4 \times 10^{16} \mathrm{~cm}^{-2}$ in that direction if there were no depletion. A mean temperature of $\sim 6 \mathrm{~K}$ along the line of sight would be compatible with the observations but is unrealistic taking into account the envelope higher temperature (10-12 K). We would be forced to put the core close to $3 \mathrm{~K}$ to prevent excess $\mathrm{C}^{18} \mathrm{O}$ emission. On top of that, the $(J: 1-0)$ line opacity would be $\sim 10$, which is not compatible with the $\mathrm{C}^{17} \mathrm{O}$ measurements. We would also have obtained $1.7 \mathrm{~K}$ for the main $\mathrm{C}^{17} \mathrm{O}$ component instead of the observed $0.4 \mathrm{~K}$. Depletion is indeed the best explanation we presently know of.

\subsection{CS depletion}

Contrarily to $\mathrm{C}^{18} \mathrm{O}$, the $\mathrm{CS}$ line is optically thick almost everywhere and the question is whether the opacity hides the dust peaks or if depletion prevents CS from revealing them. In fact, opacity is not the good reason: as Penzias (1975) has shown (see also Linke et al. 1977), if a line is optically thick 


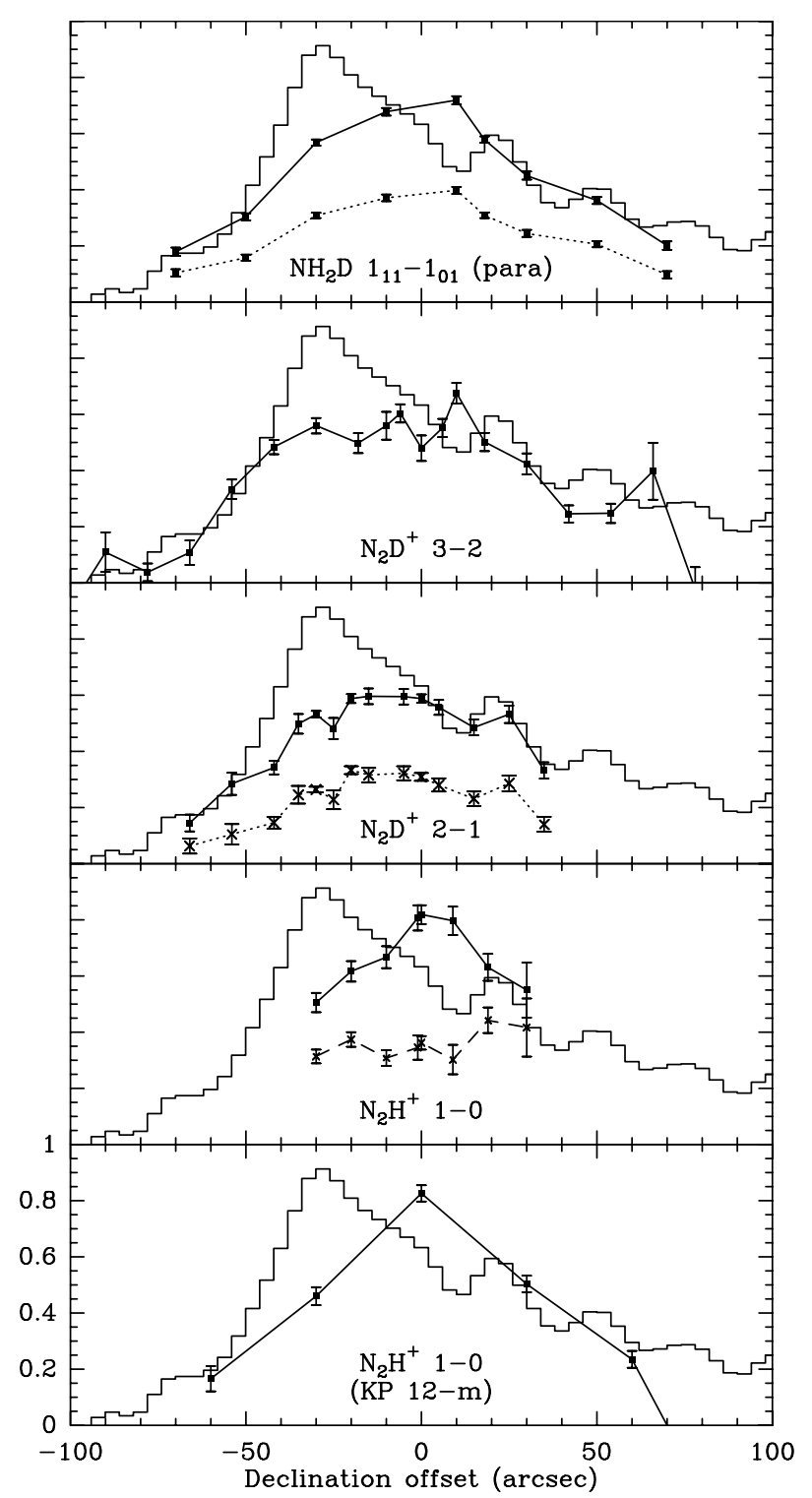

Fig. 9. North-south cut through the dust peak (RA offset $\left.=+30^{\prime \prime}\right)$. The histogram displays the $1.2 \mathrm{~mm}$ dust emission while the sets of dots trace N-bearing species through the peak. The vertical scales are all arbitrary. They are set to have the species integrated intensities proportional to the dust emission on the peak sides. Except when specified, all data come from the IRAM 30-m. For $\mathrm{NH}_{2} \mathrm{D}$ and $\mathrm{N}_{2} \mathrm{D}^{+}(J: 2-1)$, we have also plotted the sum of all the optically thin hyperfine components (dotted lines and crosses) while for $\mathrm{N}_{2} \mathrm{H}^{+}(J: 1-0)$, we have plotted the only hyperfine component $\left(\left(J_{F_{1} F}: 1_{11}-0_{01}\right)\right.$, dashed line and crosses) which remains optically thin $(\tau \leq 1)$ in this cut. Error bars are $1 \sigma$ errors on the integrated intensities.

and subthermally excited, then its intensity is merely proportional to its column density (this is illustrated in Appendix A). However, this is true only for a uniform gas slab. Here, a dilution of the peak emission in the surrounding gas is still to be expected and Penzias' prediction is only to be considered on a large scale. However, the CS peak emission is noticeably asymmetrical compared to the cloud core (Fig. 5), and a preliminary Monte-Carlo analysis has confirmed that the observed CS emission can be explained with densities lower than the

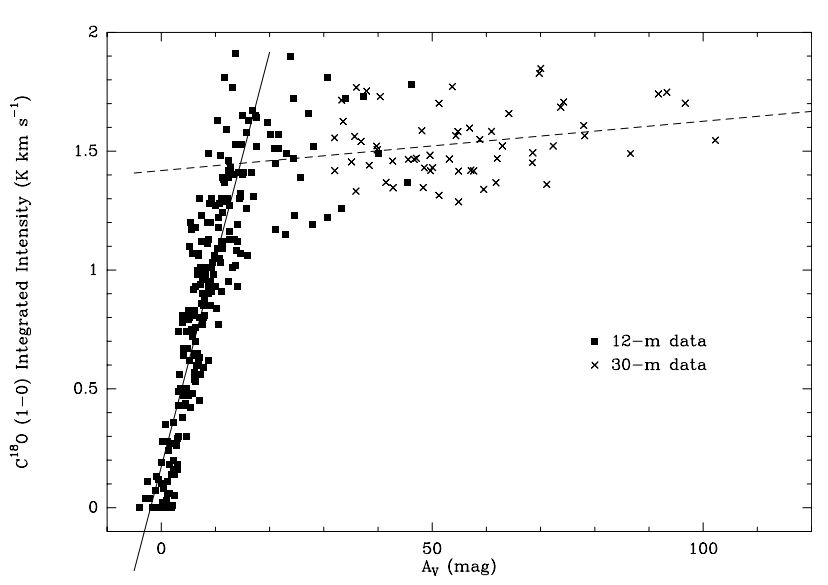

Fig. 10. $\mathrm{C}^{18} \mathrm{O}(\mathrm{J}: 1-0)$ integrated intensity pixels cross-correlated with the $A_{\mathrm{V}}$ dust map pixels from Paper II. $A_{\mathrm{V}}$ map resolution is degraded to $1^{\prime}$ for KP 12-m data and to 23" for IRAM 30-m data. The slope variation indicates that the depletion reaches at least a factor 43 in volume.

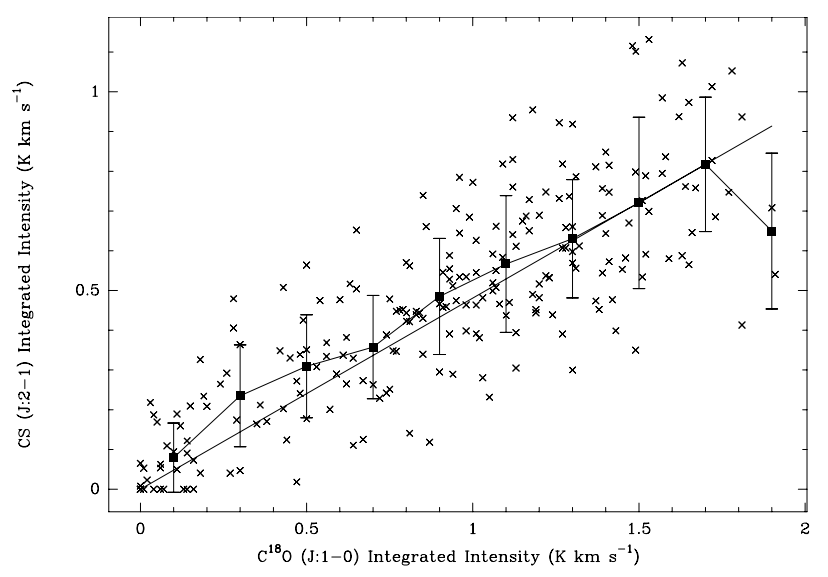

Fig. 11. $C^{32} S(J: 2-1)$ and $C^{18} O(J: 1-0)$ integrated intensity crosscorrelation. KP 12-m data only. Square symbols represent the average of the $\mathrm{C}^{32} \mathrm{~S}$ values per $0.2 \mathrm{~K} \mathrm{~km} \mathrm{~s}^{-1} \mathrm{C}^{18} \mathrm{O}$ intervals. Error bars represent the $1 \sigma$ dispersion. The straight line goes through the origin to the 9 th average value, its slope is 0.48 .

critical density (Paper I). Therefore, we think that despite its optical depth, the $\mathrm{C}^{32} \mathrm{~S}(\mathrm{~J}: 2-1)$ map is not hiding the peaks (a limited preliminary optically thin $\mathrm{C}^{34} \mathrm{~S}(\mathrm{~J}: 2-1)$ map confirms this point).

Though the CS map (Fig. 5) is somewhat different from the $\mathrm{C}^{18} \mathrm{O}$ map (Fig. 3), apart from their similar extent, their intensities are mostly proportional (Fig. 11). The proportionality factor is 0.48 and the reason is that $\mathrm{C}^{18} \mathrm{O}$ is easier to thermalize than CS. As mentioned above, this is due to the fact that CS $(J: 2-1)$ is subthermally excited and optically thick and thus its intensity is proportional to its column density. On the contrary, the $\mathrm{C}^{18} \mathrm{O}$ integrated intensity is also proportional to its column density because the $(J: 1-0)$ transition is thermalized and optically thin in most of the considered range. Therefore it is not surprising to find that CS has the same depletion behaviour as $\mathrm{C}^{18} \mathrm{O}$ (Fig. 12). CS depletion has been observed before (see, e.g., Tafalla et al. 2002). A preliminary radiative transfer analysis (Paper I) has shown that the 


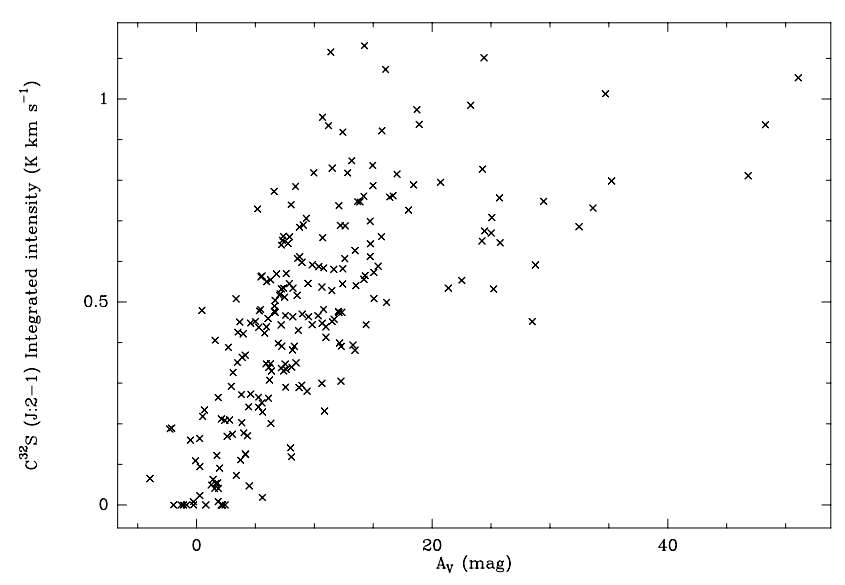

Fig. 12. $C^{32} S(J: 2-1)$ integrated intensity (KP 12-m data) crosscorrelated with $A_{\mathrm{V}}$ data degraded to $1^{\prime}$ resolution.

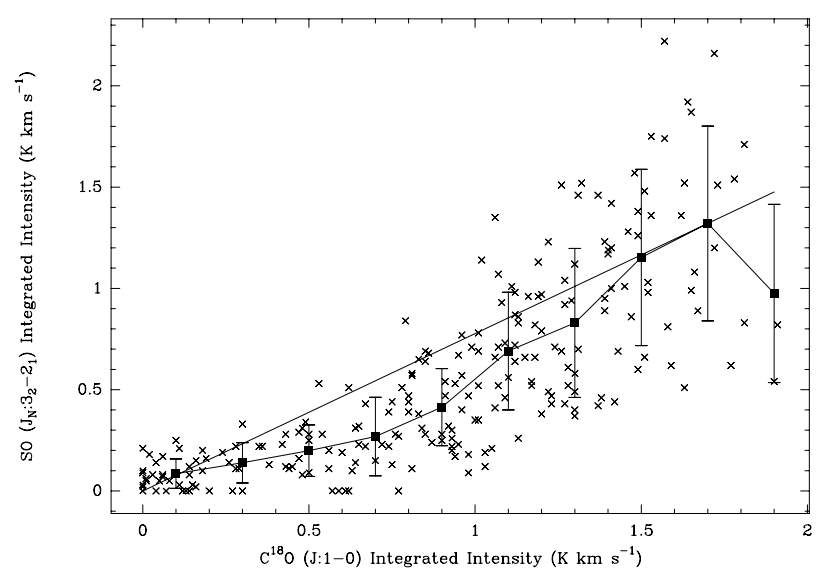

Fig. 13. ${ }^{32} \mathrm{SO}\left(J_{N}: 3_{2}-2_{1}\right)$ and $\mathrm{C}^{18} \mathrm{O}(\mathrm{J}: 1-0)$ integrated intensity crosscorrelation. KP 12-m data only. Square symbols represent the average of the ${ }^{32} \mathrm{SO}$ values per $0.2 \mathrm{~K} \mathrm{~km} \mathrm{~s}^{-1} \mathrm{C}^{18} \mathrm{O}$ intervals. Error bars represent the $1 \sigma$ dispersion. The straight line goes through the origin to the 9th average value, its slope is 0.78 .

$\mathrm{C}^{32} \mathrm{~S}$ and $\mathrm{C}^{34} \mathrm{~S}$ transitions $(J: 1-0),(J: 2-1)$ and $(J: 3-2)$ towards the $\mathrm{L} 134 \mathrm{~N}$ reference position can be explained by densities $\leq 3 \times 10^{4} \mathrm{~cm}^{-3}$, more than an order of magnitude below the CS $(J: 2-1)$ critical density $\left(n_{\mathrm{c}}=7 \times 10^{5} \mathrm{~cm}^{-3}\right)$. These findings are consistent and point to the fact that CS cannot be used as a density tracer at all in cold dark clouds because it is depleted much below its critical density. The sudden drop for $\left\langle I\left(\mathrm{C}^{18} \mathrm{O}\right)\right\rangle=1.9 \mathrm{~K} \mathrm{~km} \mathrm{~s}^{-1}$ average will be discussed in Sect. 4.4 .

\subsection{SO depletion}

Comparison of $\mathrm{SO}$ versus $\mathrm{C}^{18} \mathrm{O}$ (Fig. 13) shows that $\mathrm{SO}$ hardly appears below a $\mathrm{C}^{18} \mathrm{O}$ intensity of $0.6 \mathrm{~K} \mathrm{~km} \mathrm{~s}^{-1}$ (equivalent to $A_{\mathrm{V}}=5 \mathrm{mag}$ ) and that there is no proportionality above that limit (again, the sudden drop for $\left\langle I\left(\mathrm{C}^{18} \mathrm{O}\right)\right\rangle=1.9 \mathrm{~K} \mathrm{~km} \mathrm{~s}^{-1}$ will be discussed in Sect. 4.4). Its emission strength correlates somewhat with the C, W and S peaks (Fig. 14), but is not, or only weakly, correlated with peaks $\mathrm{N}$ and $\mathrm{E}$ which could mean that it comes closer to the $\mathrm{C}, \mathrm{W}$ and $\mathrm{S}$ peaks than CS. Interestingly enough, Bergin \& Langer (1997) find that after about 1 My of evolution, when $\mathrm{C}$ and consequently CS have already started

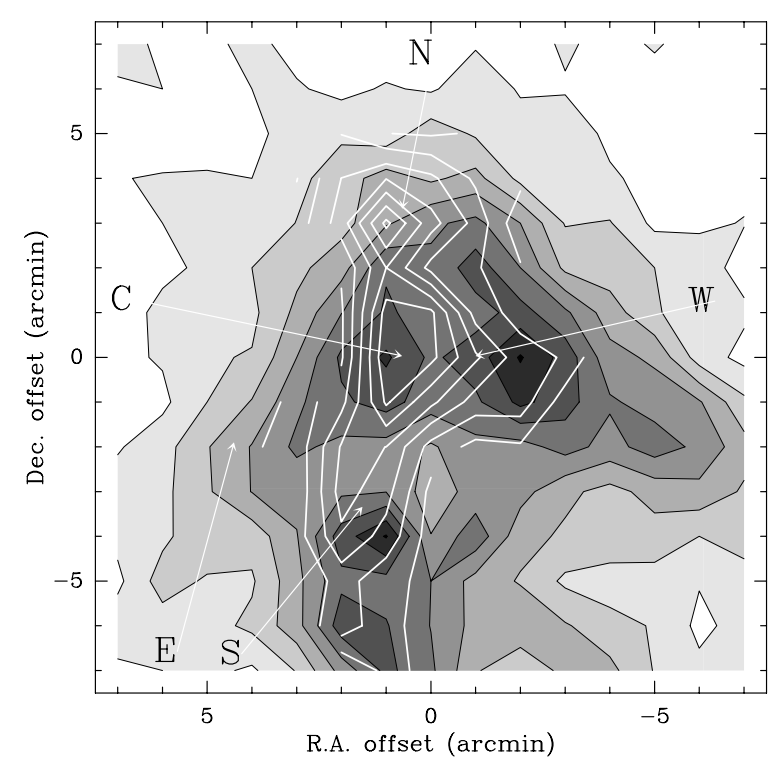

Fig. 14. ${ }^{32} \mathrm{SO}\left(J_{N}: 3_{2}-2_{1}\right)$ peak intensity (filled black contours) with $\mathrm{N}_{2} \mathrm{H}^{+}(J: 1-0)$ integrated intensity (grey contours) superimposed to it. Coordinates are offset from the reference position. ${ }^{32} \mathrm{SO}$ levels: 0.3 to 2.4 by $0.3 \mathrm{~K} . \mathrm{N}_{2} \mathrm{H}^{+}$levels: 0.4 to 2.8 by $0.4 \mathrm{~K} \mathrm{~km} \mathrm{~s}^{-1}$.

to deplete, there is a strong enhancement of SO before it starts depleting too. This enhancement has an amplitude of almost two orders of magnitude and SO eventually becomes more abundant than CS, while the model starts with a CS/SO ratio of $\sim 100$. Aikawa et al. (2003, their Figs. 3 to 5) show a similar SO enhancement in some of their depletion models as a function of core distance, though the effect does not reach the same amplitude; i.e. SO never becomes more abundant than CS but the increase of its column density as a function of radial proximity to the core center is clearly visible. We have some hints of this effect here but we can only do a preliminary analysis for the following reason: there are no SO collision coefficients yet at low temperatures $(<50 \mathrm{~K}$, Green 1994) to make a nonLTE analysis of its excitation and thus only a rotational diagram analysis is presently possible. However, due to opacity effects, as explained by Goldsmith \& Langer (1999), and to low excitation temperature ${ }^{3}$, this analysis is difficult to perform and requires many lines. We have shown that without proper corrections, SO column density would be underestimated by 2 orders of magnitude using such a diagram (Paper I). Though we presently lack data to study in detail the CS/SO column density ratio in L183, we have evaluated the CS and SO column densities towards the reference position (Paper I) for which we have observed many SO transitions and could gather a few more data from the literature. We have found $N(\mathrm{CS}) \approx 1.4 \times$ $10^{13} \mathrm{~cm}^{-2}$ and $N(\mathrm{SO}) \approx 2.5 \times 10^{14} \mathrm{~cm}^{-2}$, or a CS/SO ratio $\approx$ 0.056. In addition, CS is more extended than SO (Figs. 5 and 6) implying that away from the depleting zones, CS is probably more abundant than SO. To check this point we have compared the SO and CS integrated intensities, and the ratio of

\footnotetext{
3 As shown in Fig. 3 of Paper I, the cosmic background is no longer negligible if the excitation temperature is close to $2.73 \mathrm{~K}$, i.e. $T_{\mathrm{ex}} \leq$ $5 \mathrm{~K}$, which is the case of most species with high dipolar momentum in the typical density range of dark clouds.
} 

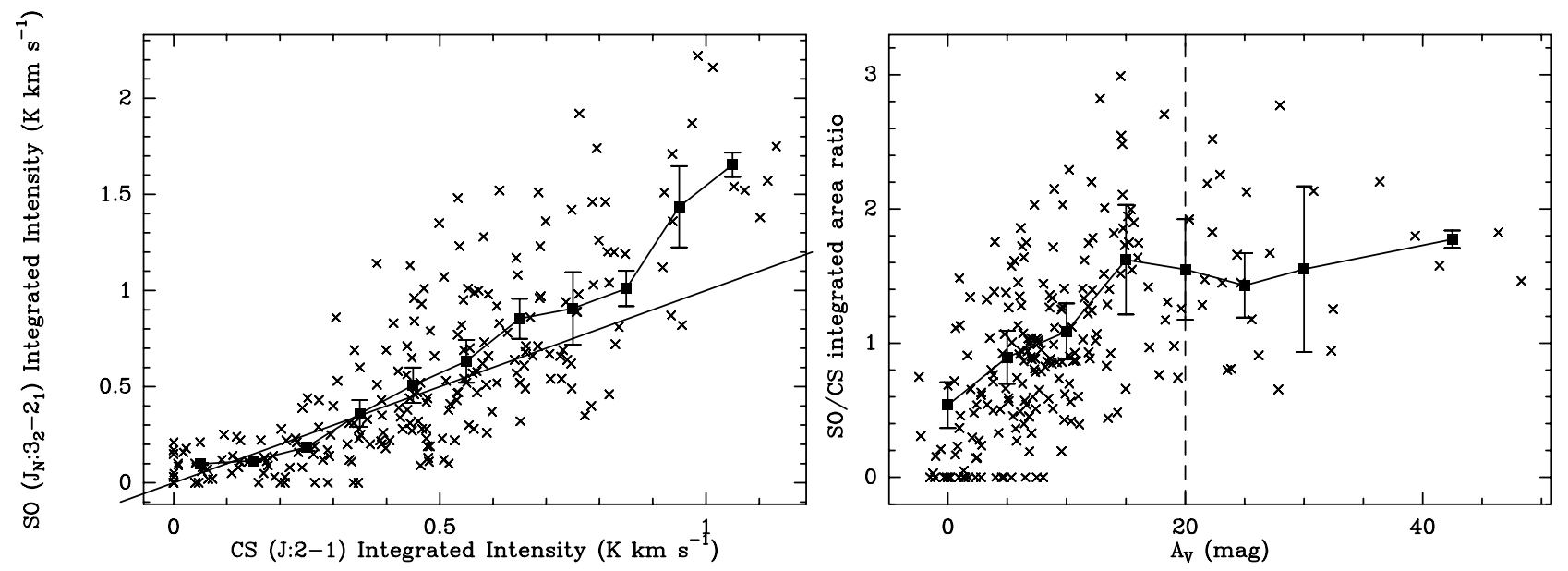

Fig. 15. Left: ${ }^{32} \mathrm{SO}\left(J_{N}: 3_{2}-2_{1}\right)$ integrated intensity cross-correlated with $\mathrm{C}^{32} \mathrm{~S}(\mathrm{~J}: 2-1)$ integrated intensity (KP $12-\mathrm{m}$ data). Square symbols represent the average of the ${ }^{32} \mathrm{SO}$ values per $0.1 \mathrm{~K} \mathrm{~km} \mathrm{~s}^{-1} \mathrm{C}^{32} \mathrm{~S}$ intervals. Error bars represent the $1 \sigma$ dispersion. The straight line is the $1: 1 \mathrm{slope}$. Right: ${ }^{32} \mathrm{SO}\left(J_{N}: 3_{2}-2_{1}\right) / \mathrm{C}^{32} \mathrm{~S}(\mathrm{~J}: 2-1)$ integrated intensity ratio cross-correlated with $A_{\mathrm{V}}$ data degraded to $1^{\prime}$ resolution. Square symbols represent the average of the $\mathrm{SO} / \mathrm{CS}$ ratio per $5 \mathrm{mag}$ intervals, except the last symbol which is the average of all the 5 points above $A_{\mathrm{V}}=32.5 \mathrm{mag}$.

SO $\left(J_{N}: 3_{2}-2_{1}\right)$ to CS $(J: 2-1)$ line integrated intensities with $A_{\mathrm{V}}$ (Fig. 15). Both lines are emitted in regions with hydrogen density below their critical density and both lines are optically thick, implying that they are both proportional to their column density to a first approximation. There is a clear trend for SO to start at a comparable or slightly lower intensity than CS and, coming closer to the depleting core, to become more intense up to $A_{\mathrm{V}}=15 \mathrm{mag}$. This obviously requires a more quantitative evaluation.

\section{4. $\mathrm{N}$-bearing species}

It has been recognized that $\mathrm{N}$-bearing species like $\mathrm{NH}_{3}$ and $\mathrm{N}_{2} \mathrm{H}^{+}$should deplete only at very high densities, $n>10^{6} \mathrm{~cm}^{-3}$ (Bergin \& Langer 1997), or even higher (e.g. $n \geq 3 \times 10^{7} \mathrm{~cm}^{-3}$ according to Aikawa et al. 2003). The reason given is the lower binding energy for nitrogen with respect to carbon or oxygen. This ability to stay longer in the gas phase should make these species good mass tracers in the dark cloud cores. Furthermore deuterated N-bearing species should be even more efficient core tracers, deuteration being enhanced by the disappearance of CO (e.g., Caselli 2002; Bacmann et al. 2003). However some question has arisen as to whether these species should eventually also start depleting in the densest, coldest pre-stellar cores as reported by Bergin et al. (2002). We have thus compared our $\mathrm{N}_{2} \mathrm{H}^{+}(J: 1-0), \mathrm{N}_{2} \mathrm{D}^{+}(J: 2-1)$ and $(J: 3-2)$ and the para$\mathrm{NH}_{2} \mathrm{D}\left(J_{K K^{\prime}}: 1_{11}-1_{01}\right)$ data of Tiné et al. (2000) to our dust map (Fig. 8).

\subsection{1. $\mathrm{N}_{2} \mathrm{H}^{+}$}

The $\mathrm{N}_{2} \mathrm{H}^{+} / A_{\mathrm{V}}$ comparison (Fig. 16a) shows that $\mathrm{N}_{2} \mathrm{H}^{+}$appears above $A_{\mathrm{V}} \approx 10$ mag only and also shows signs of depletion above $A_{\mathrm{V}} \approx 50 \mathrm{mag}$. The north-south cut across the main dust peak (Fig. 9) also suggests depletion (more correctly, the depletion concerns the parent species of $\mathrm{N}_{2} \mathrm{H}^{+}, \mathrm{N}_{2}$, as the ion cannot stick to grains itself). However the line is strongly saturated as the spectrum taken towards the dust peak shows (Fig. 17) and it could be argued that the saturation above $A_{\mathrm{V}} \approx 50$ mag in Fig. 16a comes from opacity effects. To check this point, we have studied the $\left(J_{F_{1} F}: 1_{11}-0_{01}\right)$ line and estimated its opacity using the CLASS HFS method ${ }^{4}$. We find total opacities (sum of the opacities of all the 7 hyperfine transitions of the $J: 1-0$ line) in the range $10-30$ mostly, yielding $\left(J_{F_{1} F}: 1_{11}-0_{01}\right)$ opacities in the range $0.4-1.1$. This is the weakest and the only optically thin hyperfine transition for this $(J: 1-0)$ line and this range of total opacities. We have also compared its integrated intensity to $A_{\mathrm{V}}$ (Figs. 9 and 16b). In Fig. 16b, the results are comparable to those obtained for the total integrated intensity (Fig. 16a) but the result is less convincing when we look at a few points (Fig. 9). One should note however that this particular hyperfine transition suffers from noticeable excitation temperature deviations (Caselli et al. 1995) which could explain such a difference on a few data points. In any case, the north-south cut of the $\left(J_{F_{1} F}: 1_{11}-0_{01}\right)$ transition does not reveal the dust peak and in conclusion, optical thickness cannot explain our results. Depletion of $\mathrm{N}_{2}$ is the only possibility left.

To evaluate the range of densities where $\mathrm{N}_{2} \mathrm{H}^{+}$is present, we can try to analyze the $\mathrm{N}_{2} \mathrm{H}^{+}$spectra with CLASS HFS method combined with a crude non-LTE (LVG) analysis of the physical conditions, using Green's collision coefficients (which do not take into account the hyperfine splitting, Green 1975). In the case of the spectrum taken towards the dust peak (Fig. 17), we get a total opacity of $\sim 20$ (i.e. individual hyperfine component opacities from 0.8 to 5 ), an excitation temperature of $4.6 \mathrm{~K}$ and a remarkably narrow linewidth $\left(0.18 \mathrm{~km} \mathrm{~s}^{-1}\right)$. Considering a single hyperfine transition as an isolated line, the LVG model indicates a local density of $\sim 2.5 \times 10^{4} \mathrm{~cm}^{-3}$ and a total column density of $\sim 10^{13} \mathrm{~cm}^{-2}$ if we suppose the kinetic temperature to be $10 \mathrm{~K}$. If we try to fit the $\left(J_{F_{1} F}: 1_{11}-0_{01}\right)$ transition, we find a density $\sim 8 \times 10^{4} \mathrm{~cm}^{-3}$ instead, and towards

\footnotetext{
4 The Grenoble data reduction package. The HFS method assumes LTE holds among the hyperfine components which is approximate in the case of $\mathrm{N}_{2} \mathrm{H}^{+}(\mathrm{J}: 1-0)$ (Caselli et al. 1995).
} 

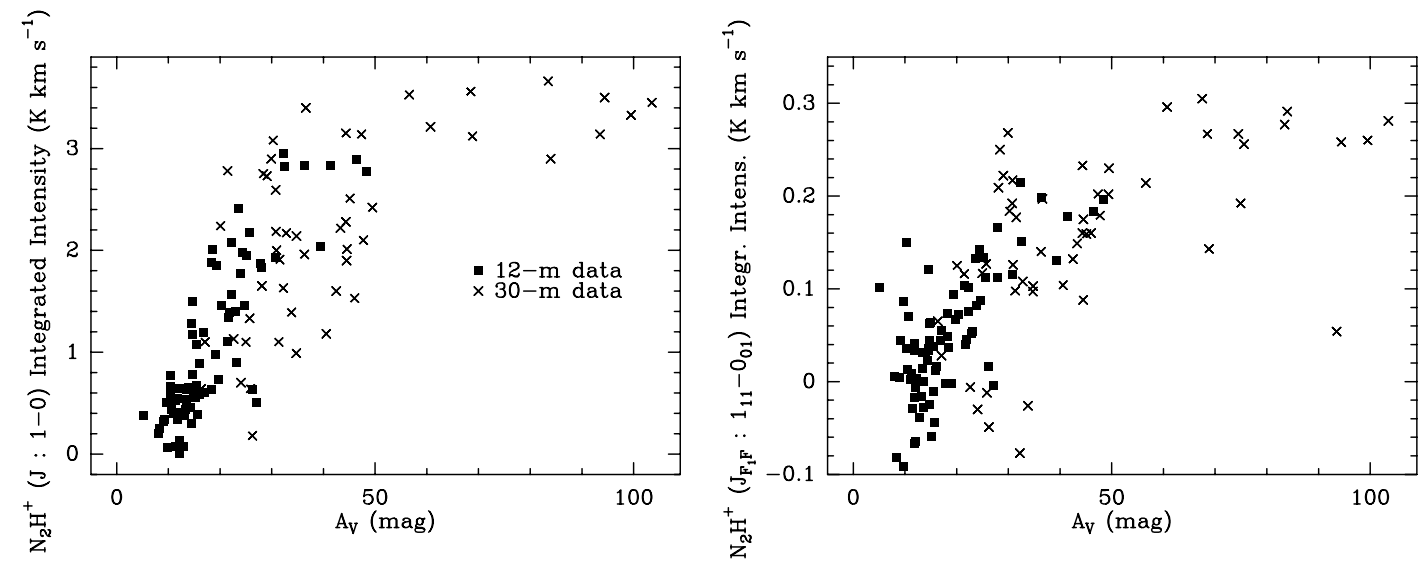

Fig. 16. a), b) $\mathrm{N}_{2} \mathrm{H}^{+}(J: 1-0)$ integrated intensity pixels cross-correlated with the $A_{\mathrm{V}}$ dust map pixels from Paper II; a) (left) All seven hyperfine components have been included; b) (right) only the weakest, optically thin $\left(J_{F_{1} F}: 1_{11}-0_{01}\right)$ component has been included. $A_{\mathrm{V}}$ map resolution is degraded to $1^{\prime}$ for KP 12-m data and to 23" for IRAM 30-m data.

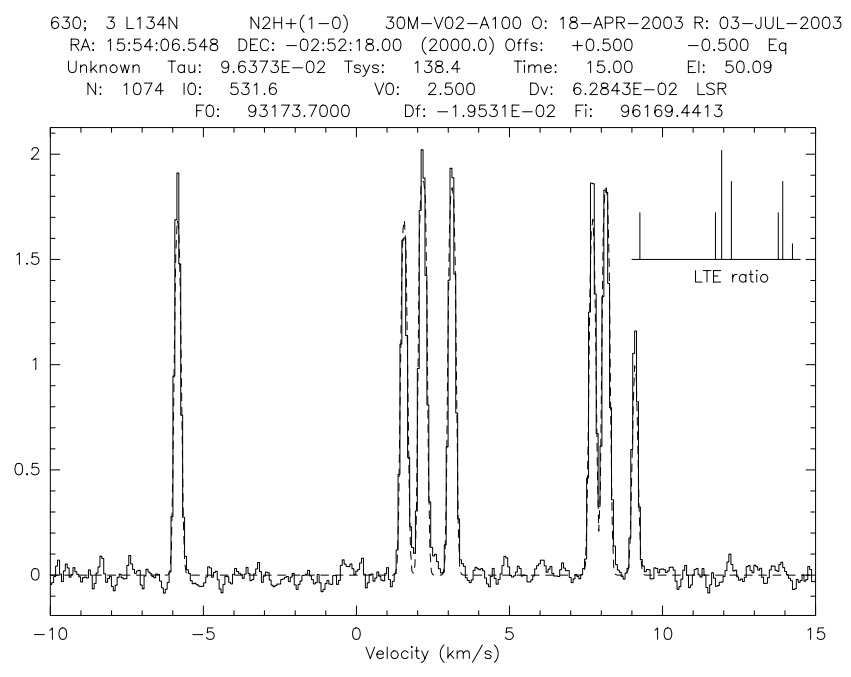

Fig. 17. $\mathrm{N}_{2} \mathrm{H}^{+}(J: 1-0)$ spectrum taken towards the dust peak with the IRAM 30-m. The dashed curve is the CLASS hyperfine Gaussian fit result, the (1:3:5:7) LTE ratio of the hyperfine components is shown in the top right corner.

other places in the cloud, where the $\mathrm{N}_{2} \mathrm{H}^{+}$line is not so optically thick, the same crude LVG analysis yields densities around $10^{5} \mathrm{~cm}^{-3}$. It thus indicates that towards the main dust core we are mostly measuring the outer parts of the $\mathrm{N}_{2} \mathrm{H}^{+}$region where the density is low. This is due to the fact that the escaping photons are those emitted in the last absorbing layer outside the core, the one with the lowest density as the core density decreases radially (Paper II). This density of $2.5 \times 10^{4} \mathrm{~cm}^{-3}$ is consistent with the density of $3 \times 10^{4} \mathrm{~cm}^{-3}$ we have found for $\mathrm{C}^{18} \mathrm{O}$ and $\mathrm{CS}$ (Paper I) together with the fact that $\mathrm{CO}$ and $\mathrm{N}_{2} \mathrm{H}^{+}$ cannot coexist: $\mathrm{N}_{2} \mathrm{H}^{+}$appears where $\mathrm{CO}$ disappears. Towards the center, it is difficult to evaluate precisely at which density the ion starts to disappear by depletion but the turn-over point at $A_{\mathrm{V}} \approx 50$ mag in Fig. 16 corresponds to a density of $\sim 4 \times$ $10^{5} \mathrm{~cm}^{-3}$ in Fig. 3 of Paper II. Because the dust peak maximum density is $2 \times 10^{6} \mathrm{~cm}^{-3}$, we can, conservatively, claim that $\mathrm{N}_{2} \mathrm{H}^{+}$depletion starts below or close to $10^{6} \mathrm{~cm}^{-3}$ and probably up to a factor of 2 lower.

\subsection{2. $\mathrm{N}_{2} \mathrm{D}^{+}$and $\mathrm{NH}_{2} \mathrm{D}$}

Another way to check the opacity problem is to look at the rarer isotopomer $\mathrm{N}_{2} \mathrm{D}^{+}$. Interestingly enough, the $\left[\mathrm{N}_{2} \mathrm{D}^{+}\right] /\left[\mathrm{N}_{2} \mathrm{H}^{+}\right]$ abundance ratio is very high towards molecular peak $\mathrm{C}, \approx 0.35$ (Tiné et al. 2000). This large deuteration enhancement has been seen for many species in $\mathrm{CO}$ depleted cores (including $\mathrm{ND}_{3}$ in $\mathrm{B} 1$, Lis et al. 2002; and $\mathrm{CD}_{3} \mathrm{OH}$ in IRAS 16293-2422, Parise et al. 2004) and is clearly linked to the large depletion of heavy species (see, e.g., Roberts et al. 2003).

Despite its relatively high abundance, the $\mathrm{N}_{2} \mathrm{D}^{+}$high electric dipole moment makes it difficult to populate the $J=2$ rotational level of this species in cold, moderately dense dark cloud cores and thus the $(J: 3-2)$ transition is probably optically thin or close to optical thinness in the present source. Because the case is not so straightforward for the $(J: 2-1)$ transition and for the para- $\mathrm{NH}_{2} \mathrm{D}\left(J_{K K^{\prime}}: 1_{11}-1_{01}\right)$ transition, we have selected optically thin hyperfine components of both species to see if the maps were different and if we could find the dust peak. We find that the maps of the selected hyperfine components are identical (within the noise) to the maps presented here (Fig. 8). This can be seen in Fig. 9 where the optically thin component integrated intensities trace the same profile as the total integrated intensities for these two lines. In Fig. 8, we see the molecular peak $30^{\prime \prime}$ north of the dust peak and a small extension to the dust peak for both $\mathrm{NH}_{2} \mathrm{D}$ and $\mathrm{N}_{2} \mathrm{D}^{+}$but the dust peak is clearly absent. Thus it does not seem possible to find any species to trace the dust peak except $\mathrm{H}_{2} \mathrm{D}^{+}$which has been recently discovered in L1544 (Caselli et al. 2003) and also towards this source (Caselli et al. in preparation) and, with even greater observing difficulties, $\mathrm{D}_{2} \mathrm{H}^{+}$, recently discovered in $16293 \mathrm{E}$ (Vastel et al. 2004).

From the molecular peak to the dust peak, the dust column density increases by a factor of $\sim 1.5$ and the peak density by a factor of $\sim 5$ following the density profile of Paper II (their Fig. 3). In first approximation, the intensity of the line is proportional to its column density (CD) and to the gas density, $n\left(\mathrm{H}_{2}\right)$, as long as the gas density is below the critical density for the transition we observe. This has been checked with an LVG model for the $\mathrm{N}_{2} \mathrm{D}^{+}(J: 3-2)$ line and the range of 
densities implied here. The intensity of the line is thus proportional to the integral along the line of sight of the product $\mathrm{CD} \times n\left(\mathrm{H}_{2}\right)$. This integral increases by a factor $\sim 4$ from the molecular peak to the dust peak, while the $\mathrm{N}_{2} \mathrm{D}^{+}(J: 3-2)$ line integrated intensity is approximately constant. Therefore, the line hyperfine components being close to optical thinness, we must conclude that the line of sight depletion reaches $\sim 4$ compared to the molecular peak. Consequently, the volume depletion must be at least a factor of $\sim 15$ for these $\mathrm{N}$-bearing species on average if we suppose that we may have missed a $20 \%(3 \sigma)$ intensity increase from molecular peak to dust peak in our observations (we expect a factor 4 increase and we may have only $1.2,(4-1) /(1.2-1)=15$, the -1 is due to the undepleted part in front and behind the core $)^{5}$.

Instead of depletion, one could argue that the molecular peak is peculiar with a strong $\mathrm{N}_{2} \mathrm{D}^{+}$enrichment. In fact, Fig. 9 reveals that $\mathrm{N}_{2} \mathrm{D}^{+}$and $\mathrm{NH}_{2} \mathrm{D}$ do follow the dust profile on both sides of the dust peak and that the molecular peak existence and asymmetry with respect to the dust peak are due in fact to the asymmetric dust profile itself. The molecular peak lies on the northern ridge (Pagani et al. 2003; Paper II) at the closest position to the core before depletion sets in. Because of the elongated ridge with a slow density variation, the region of high density without depletion is large enough to stand out as a molecular peak. In all the other directions away from the dust peak, the density drops much faster and therefore the region of maximum density without depletion is too narrow to be revealed. The column density has somewhat the same effect but to a lesser extent as we have seen above (the density changed by a factor 5 while the column density by a factor 1.5 between molecular peak $\mathrm{C}$ and dust peak 1). Therefore, the molecular peak is indeed displaced to the north by $30^{\prime \prime}$ due to depletion.

\subsection{Depletion trigger}

Until now, it has not been clear whether the main trigger for depletion of species such as CO and CS is dust opacity (i.e. protection against evaporation by UV radiation) or density (i.e. speed of depletion). It seems from this study that density is the major trigger as the most detailed studies published today quote densities of the order a few $10^{4} \mathrm{~cm}^{-3}$ very close to our own value of $3 \times 10^{4} \mathrm{~cm}^{-3}$ which is the density we have found in Paper I to explain $\mathrm{C}^{18} \mathrm{O}, \mathrm{C}^{32} \mathrm{~S}$ and $\mathrm{C}^{34} \mathrm{~S}$ line intensities towards the reference position. Tafalla et al. (2002) find $3-6 \times$ $10^{4} \mathrm{~cm}^{-3}$, Tafalla et al. (2004) find 2.5 and $7.8 \times 10^{4} \mathrm{~cm}^{-3}$, and Jørgensen et al. (2004) find $3 \times 10^{4} \mathrm{~cm}^{-3}$. On the contrary optical extinction thresholds are usually found close to $A_{\mathrm{V}}=$ 10 mag (see Sect. 4.1) while we report $A_{\mathrm{V}}=20$ mag here (and this is not even sufficient for peak 5-peak E which shows no depletion). Moreover we have recently found in a cloud in the Taurus region $\mathrm{C}^{18} \mathrm{O}$ depletion starting at $A_{\mathrm{V}} \sim 4 \mathrm{mag}$, the lowest $A_{\mathrm{V}}$ value presently known for $\mathrm{CO}$ depletion (Boudet et al., in preparation).

\footnotetext{
5 A more accurate analysis will be performed in a coming paper when the detailed collisional coefficients (including hyperfine structure) of $\mathrm{N}_{2} \mathrm{H}^{+}$will be completely computed (Daniel et al. 2003; Daniel et al. in preparation).
}

Concerning the two important nitrogen-bearing species $\left(\mathrm{N}_{2} \mathrm{H}^{+}\right.$and $\mathrm{NH}_{3}$, the latter being represented by its deuterated isotopomer $\mathrm{NH}_{2} \mathrm{D}$ only), we find that they are depleted at densities somewhere between $5 \times 10^{5}$ and $10^{6} \mathrm{~cm}^{-3}$. While Bergin \& Langer (1997) predict such an effect at this density level for grains covered by polar $\left(\mathrm{H}_{2} \mathrm{O}\right)$ ices, a more recent paper (Aikawa et al. 2003) estimates that $\mathrm{N}_{2} \mathrm{H}^{+}$and $\mathrm{NH}_{3}$ depletion is unlikely below densities of $3 \times 10^{7} \mathrm{~cm}^{-3}$. The possible reason which they invoke for this effect is that the binding energy of $\mathrm{N}_{2}$ onto grains could be lower than usually advocated (a similar effect is found by Bergin \& Langer 1997, for grains with no ice or apolar (CO) ice). The present result would tend to show that the polar ice case described by Bergin \& Langer (1997) is probably closer to the truth.

\subsection{The peak E case}

Despite a peak visual extinction of $\sim 25$ mag (Paper II), Peak E shows no $\mathrm{CO}$ depletion and correlatively very weak $\mathrm{N}_{2} \mathrm{H}^{+}$emission. This peak is in fact the $\mathrm{C}^{17} \mathrm{O}$ and $\mathrm{C}^{18} \mathrm{O}$ emission peak of the whole cloud. This peak is also a 100 and $200 \mu \mathrm{m}$ peak (Paper I) from which we have derived an average temperature of $\sim 15 \mathrm{~K}$. However, this temperature yields a $200 \mu$ m-derived dust extinction $A_{\mathrm{V}} \approx 7$ mag (Paper I) too low compared to the NIR-determined visual extinction (Paper II). This probably indicates that the bulk of the dust is at a lower temperature and that some layers are at higher temperatures than $15 \mathrm{~K}$. These warm layers would not allow depletion of apolar molecules like $\mathrm{CO}$ but their total opacity being low, they cannot account for all the $\mathrm{C}^{18} \mathrm{O}$ emission detected. In Paper I, we have tried to fit the $\mathrm{C}^{18} \mathrm{O}(J: 1-0),(J: 2-1)$ and $(J: 3-2)$ rotational line emission with a Monte-Carlo code. Although we somewhat failed to reproduce the very low $(J: 2-1)$ line emission, we are probably close to the solution, in terms of the column density estimate which is $3.5 \times 10^{15} \mathrm{~cm}^{-2}$. Compared to a peak $A_{\mathrm{V}}$ of $\sim 25 \mathrm{mag}$, this yields an abundance of $\sim 1.4 \times$ $10^{-7}$, which is the standard value recommended by Frerking et al. (1982). We have only weak $C^{32} S(J: 2-1)$ line detections towards peak $\mathrm{E}$ in sharp contrast with $\mathrm{C}^{18} \mathrm{O}$ intensity. Though it is yet difficult to derive a density from a single CS transition, keeping the same $\mathrm{C}^{32} \mathrm{~S} / \mathrm{C}^{18} \mathrm{O}$ abundance ratio as measured towards the reference position (i.e. $\sim 0.005)$ we find that $\mathrm{C}^{32} \mathrm{~S}$ $(J: 2-1)$ and $\mathrm{C}^{18} \mathrm{O}(J: 1-0),(J: 2-1)$ and $(J: 3-2)$ are reasonably compatible with a core density of $\sim 10^{4} \mathrm{~cm}^{-3}$. Thus, density could again be the explanation for the absence of depletion towards Peak E. Why this peak is less dense and (partially but substantially) warmer than the other peaks in this cloud is not yet clear.

In both Figs. 11 and 13, there is a drop (-30\%) of intensity with respect to $\mathrm{C}^{18} \mathrm{O}$ for the last averaged point (corresponding to the strongest $\mathrm{C}^{18} \mathrm{O}$ emission). This average point contains only $\mathrm{C}^{18} \mathrm{O}$ positions in the direction of peak $\mathrm{E}$. As we have seen above, the $\mathrm{C}^{18} \mathrm{O}$ abundance is normal towards peak E, and we could find the same $\mathrm{C}^{32} \mathrm{~S} / \mathrm{C}^{18} \mathrm{O}$ abundance ratio as measured towards the reference position provided that the peak density be no higher than $\sim 10^{4} \mathrm{~cm}^{-3}$. Though we cannot do a similar excitation analysis for $\mathrm{SO}$, it is most 
probable that the intensity drop is also explained by the sole density factor. Thus the peak $\mathrm{E}$ is probably not peculiar regarding the species abundances. The question then arises of whether the weak $\mathrm{N}_{2} \mathrm{H}^{+}$line observed in that direction is due to chemical destruction as predicted by models or only to the fact that the density is so low that it cannot be observed. The $\mathrm{N}_{2} \mathrm{H}^{+}$line we have detected is so weak $(\sim 0.15 \mathrm{~K}(4 \sigma))$ that only the strongest component $\left(J_{F_{1} F}: 1_{23}-0_{12}\right)$ emerges from the noise and no CLASS HFS analysis can be done. However, we know the density to be probably no more than $10^{4} \mathrm{~cm}^{-3}$ and therefore we can use the $L V G$ code to crudely estimate the $\mathrm{N}_{2} \mathrm{H}^{+}$column density. With $T_{\text {kin }}=10 \mathrm{~K}$, we find a $\left(J_{F_{1} F}: 1_{23}-0_{12}\right)$ line column density of $3 \times 10^{11} \mathrm{~cm}^{-2}$ which yields a total column density of $\sim 1.2 \times 10^{12} \mathrm{~cm}^{-2}$. Compared to the dust extinction of $25 \mathrm{mag}$ towards peak \#5, we find a $\mathrm{N}_{2} \mathrm{H}^{+}$abundance of $5 \times 10^{-11}$. If we repeat the same analysis for a few $\mathrm{N}_{2} \mathrm{H}^{+}$observations towards lines of sight with $\sim 40$ mag of extinction (to stay away from both the depletion threshold and the threshold of destruction by $\mathrm{CO}$ ), we find an abundance of $1.2-2.1 \times 10^{-10}, 2$ to 4 times higher than towards peak $\mathrm{E}$. Therefore, a low volume density is not the unique reason for the quasi-absence of $\mathrm{N}_{2} \mathrm{H}^{+}$emission towards peak E. It has also an abundance lower than in the $\mathrm{CO}$ depleted region. Several theoretical and observational studies have shown that $\mathrm{N}_{2} \mathrm{H}^{+}$appears in the gas when $\mathrm{CO}$ depletes (Bergin \& Langer 1997; Bergin et al. 2001; Caselli 2002; Aikawa et al. 2003; Jørgensen et al. 2004). This analysis would appear to again confirm this effect.

\section{Conclusions}

All species containing at least one atom of the CNO group are depleted in the most inner core of L183. CO, CS and SO are largely depleted but their depletion starts only at a relatively high optical extinction $\left(A_{\mathrm{V}} \approx 20 \mathrm{mag}\right)$ unseen elsewhere. On the contrary, the density $\left(n \approx 3 \times 10^{4} \mathrm{~cm}^{-3}\right)$ for which depletion starts is similar to other findings and this result points towards the fact that density is the main parameter to trigger depletion rather than protection against UV radiation. Depletion effects, reported now in many dark clouds, prevent $\mathrm{CO}$ from being an accurate mass tracer for cold dense cores, and forbid the search of such cores via its rarer isotopomers $\left(\mathrm{C}^{17} \mathrm{O}, \mathrm{C}^{18} \mathrm{O}\right)$. Similarly, CS is not a good density tracer in many cold clouds because it survives in the gas phase only at densities much below its critical density and thus its intensity is mostly proportional to its column density. $\mathrm{N}$-bearing species (especially $\mathrm{N}_{2} \mathrm{H}^{+}$and $\mathrm{NH}_{3}$ ) are better tracers of dense cores but only up to densities below $10^{6} \mathrm{~cm}^{-3}$. Beyond that limit, they also disappear (Bergin et al. 2001). Like for L1544 and 16293E, only $\mathrm{H}_{2} \mathrm{D}^{+}$(Caselli et al. 2003) and $\mathrm{D}_{2} \mathrm{H}^{+}$(Vastel et al. 2004) should be observable in the inner core (Caselli et al., in preparation).

Finally, species abundances for chemical models such as those provided by Dickens et al. (2000) for L183 are meaningless averages along the line of sight of species which are not necessarily coexistent spatially. Only models including geometry, depletion and radiation transport can nowadays be usefully compared to the detailed observations allowed by modern telescopes. Such models have recently been proposed by Bergin et al. (2001, including a chemical network scheme) or by Jørgensen et al. (2004) and similar studies in protostellar cores have been presented by Tafalla et al. (2002, 2004).

Acknowledgements. J. R. Pardo's research work is supported by Spanish MCyT grants ESP 2003-01627 and AYA 2002-10113-E. We thank the referee, E. D. (Ted) Bergin, for useful comments and suggested improvements to the paper.

\section{References}

Aikawa, Y., Ohashi, N., \& Herbst, E. 2003, ApJ, 593, 906

Alves, J., Lada, C. J., \& Lada, E. A. 1999, ApJ, 515, 265

Bacmann, A., Lefloch, B., Ceccarelli, C., et al. 2003, ApJ, 585, L55

Bergin, E. A., \& Langer, W. D. 1997, ApJ, 486, 316

Bergin, E. A., Ciardi, D. R., Lada, C. J., Alves, J., \& Lada, E. A. 2001, ApJ, 557, 209

Bergin, E. A., Alves, J., Huard, T., \& Lada, C. J. 2002, ApJ, 570, L101

Caselli, P., Myers, P. C., \& Thaddeus, P. 1995, ApJ, 455, L77

Caselli, P. 2002, P\&SS, 50, 1133

Caselli, P., van der Tak, F., Ceccarelli, C., \& Bacmann, A. 2003, A\&A, 403, L37

Daniel, F., Dubernet, M.-L., Pagani, L. 2003, in SF2A Scientific Highlights 2003, ed. F. Combes, D. Barret, T. Contini, \& L. Pagani (Les Ulis: EDP Sciences), 205

Dickens, J. E., Irvine, W. M., Snell, R. L., et al. 2000, ApJ, 542, 870

Frerking, M. A., Langer, W. D., \& Wilson, R. W. 1982, ApJ, 262, 590

Goldsmith, P., \& Langer, W. D. 1999, ApJ, 517, 219

Green, S. 1975, ApJ, 201, 366

Green, S. 1994, ApJ, 434, 188

Jørgensen, J. K., Schöier, F. L., \& van Dishoeck, E. F. 2004, A\&A, in press

Juvela, M., Mattila, K., Lehtinen, K., et al. 2002, A\&A, 382, 583

Kramer, C., Alves, J., Lada, C. J., et al. 1999, A\&A, 342, 257

Kutner, M. L., \& Ulich, B. L. 1981, ApJ, 250, 341

Linke, R. A., Goldsmith, P. F., Wannier, P. G., Wilson, R. W., \& Penzias, A. A. 1977, ApJ, 214, 50

Lis, D. C., Roueff, E., Gerin, M., et al. 2002, ApJ, 571, L55

Pagani, L., Gallego, A. T., \& Apponi, A. J. 2001, A\&A, 380, 384 (Erratum: 2002, A\&A, 381, 1094)

Pagani, L., Pardo, J.-R., Fich, M., Motte, F., \& Stepnik, B. 2002, in Infrared and Submillimeter Space Astronomy, EAS Series, Vol. 4, ed. M. Giard et al., held in Toulouse, June 2001 (Les Ulis: EDP Sciences), 145 (Paper I)

Pagani, L., Lagache, G., Bacmann, A., et al. 2003, A\&A, 406, L59

Pagani, L., Bacmann, A., Motte, F., et al. 2004, A\&A, 417, 605 (Paper II)

Parise, B., Castets, A., Herbst, E., et al. 2004, A\&A, 416, 159

Penzias, A. A. 1975, in Atomic and molecular physics and the interstellar matter; Proceedings of the Twenty-sixth Summer School of Theoretical Physics, Les Houches, France, July 1-August 23, 1974 (Amsterdam; North-Holland Publishing Co.), 373

Roberts, H., Herbts, E., \& Millar, T. J. 2003, ApJ, 591, L41

Swade, D. A. 1989a, ApJS, 71, 219

Swade, D. A. 1989b, ApJ, 345, 828

Tafalla, M., Myers, P. C., Caselli, P., Walmsley, C. M., \& Comito, C. 2002, ApJ, 569, 815

Tafalla, M., Myers, P. C., Caselli, P., \& Walmsley, C. M. 2004, A\&A, 416, 191

Teyssier, D., Hennebelle, P., \& Pérault, M. 2002, A\&A, 382, 624

Tiné, S., Roueff, E., Falgarone, E., Gerin, M., \& Pineau des Forêts, G. 2000, A\&A, 356, 1039

Vastel, C., Phillips, T. G., \& Yoshida, H. 2004, ApJ, 606, L127

Willacy, K., Langer, W. D., \& Velusamy, T. 1998, ApJ, 507, L171 


\section{Online Material}




\section{Appendix A: Subthermally excited optically thick lines}

A radiotelescope which observes a source with opacity $\tau$ and excitation temperature $T_{\text {ex }}$ by comparison with an empty field, detects an antenna temperature $T_{\mathrm{a}}$ increase given by

$\Delta T_{\mathrm{a}}=\eta\left(1-\mathrm{e}^{-\tau}\right)\left(J\left(T_{\mathrm{ex}}\right)-J\left(T_{\mathrm{bg}}\right)\right)$

with $\eta$ the coupling efficiency of the telescope, $T_{\mathrm{bg}}$, the temperature of the cosmological background radiation and $J(\mathrm{~T})$ is given by

$J(T)=\frac{h v}{k} \frac{1}{\mathrm{e}^{\frac{h v}{k T}}-1}$

where $h$ is the Planck constant, $k$ the Boltzmann constant and $v$ the line frequency. When $\tau \gg 1$, Eq. (A.1) simplifies to

$\Delta T_{\mathrm{a}}=\eta\left(J\left(T_{\mathrm{ex}}\right)-J\left(T_{\mathrm{bg}}\right)\right)$

which would seem to be independent of $\tau$ and therefore of the species column density as $\tau$ is defined by

$\tau=\frac{c^{2} g_{\mathrm{u}} A_{\mathrm{ul}} N_{1}}{8 \pi g_{\mathrm{l}} v^{2} \Delta v}\left[1-\mathrm{e}^{-\frac{h \nu}{k T_{\mathrm{ex}}}}\right]$

with $c$ the light speed, $A_{\mathrm{ul}}$ the Einstein spontaneous emission coefficient, $g_{\mathrm{u}}$ and $g_{1}$ the upper and lower species level statistical weights and $N_{1}$, the lower level column density. The latter is related to the total column density via the partition function.

In fact, when a line is optically thick and the local density is much below the critical density (defined by $n_{\text {crit }}=A_{\mathrm{ul}} / \gamma_{\mathrm{ul}}$, $\gamma_{\mathrm{ul}}$ being the collision probability), each exciting collision has a higher probability to be followed by a radiative decay rather than a collisional deexcitation. But similarly, if the photon is reabsorbed because of high opacity, it will still be reemitted rather than having the species be deexcited by a collision. Thus each exciting collision will give birth to a photon which will eventually escape. If the opacity is high, many photons are retained in the cloud before escaping and the net result is an increase of the excitation temperature and therefore of the antenna temperature in Eq. (A.3). We refer the reader to Penzias (1975) or Linke et al. (1977) for a mathematical demonstration. Here we want to illustrate this result via two simulations and actual observations.

Figures A.1 and A.2 show the case of $\mathrm{C}^{32} \mathrm{~S}(\mathrm{~J}: 1-0)$ and $(J: 2-1)$ lines in a very low density medium $\left(10^{3} \mathrm{~cm}^{-3}\right)$ and at two different kinetic temperatures (Fig. A.1, $T_{\text {kin }}=10 \mathrm{~K}$, Fig. A.2, $T_{\text {kin }}=50 \mathrm{~K}$ ). The line opacity and antenna temperatures are proportional to the total column density below $N=$ $10^{13} \mathrm{~cm}^{-2}$, which corresponds to $\tau \lesssim 1$ and $T_{\text {ex }}$ is almost constant. Above $N=10^{13} \mathrm{~cm}^{-2}$, i.e. $\tau>1$, the excitation temperature starts to rise and the antenna temperature keeps increasing linearly first then deviates while approaching the excitation limit (collisional deexcitation starts to be efficient when too many molecules are in the excited state).

In $\mathrm{L} 183$, the $\mathrm{N}_{2} \mathrm{H}^{+}(\mathrm{J}: 1-0)$ line has the advantage of having both a hyperfine transition always optically thin $\left(J_{F_{1} F}: 1_{11}-0_{01}\right)$ and another one, almost always optically thick $\left(J_{F_{1} F}: 1_{23}-0_{12}\right)$ in the range of conditions offered by this cloud. The line has a

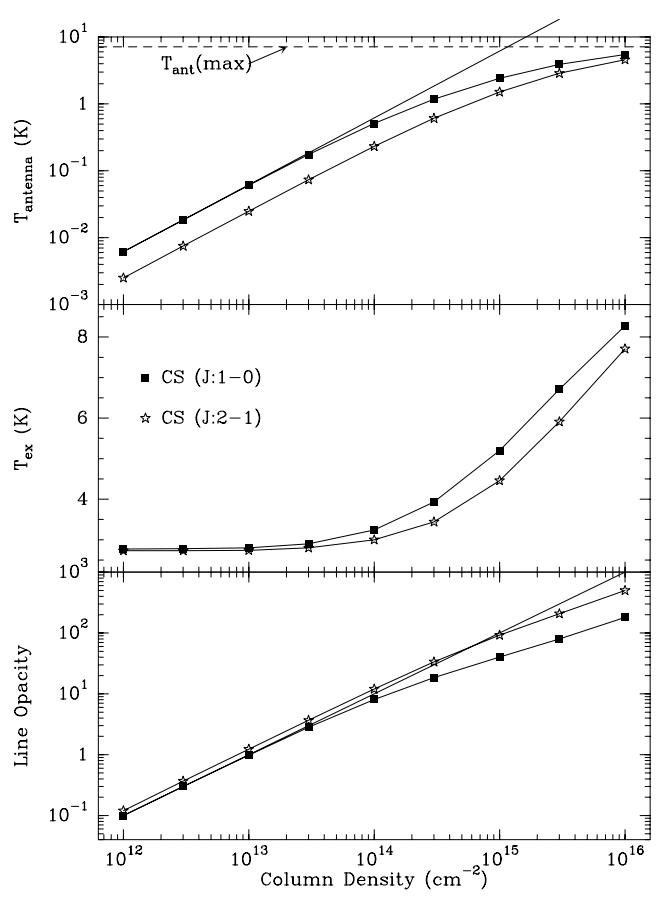

Fig. A.1. $C^{32} S(J: 1-0)$ and $(J: 2-1)$ transition properties as a function of column density. LVG model with $T_{\text {kin }}=10 \mathrm{~K}, \delta V=1 \mathrm{~km} \mathrm{~s}^{-1}$ and $n=10^{3} \mathrm{~cm}^{-3}$.

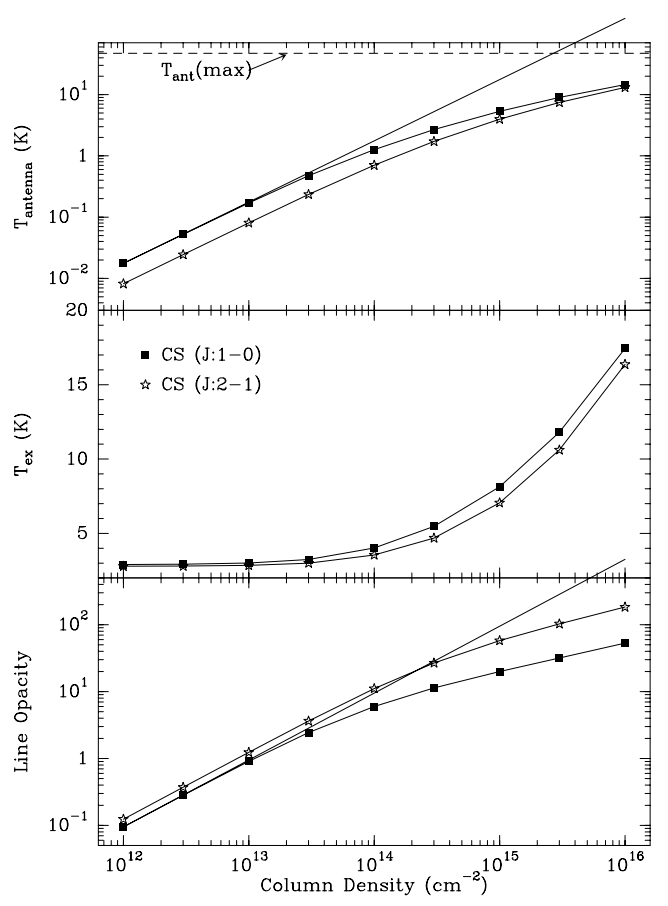

Fig. A.2. Same as Fig. A.1 except $T_{\text {kin }}=50 \mathrm{~K}$.

critical density around $10^{6} \mathrm{~cm}^{-3}$ and is therefore subthermally excited everywhere in the cloud. Figure A.3 shows the comparison of the two hyperfine transition integrated intensities together with the 1:7 slope corresponding to their LTE ratio. The proportionality is clearly visible despite a slight departure from the 1:7 slope. This departure is probably due to the emergence of a saturation effect or to the fact that the $\left(J_{F_{1} F}: 1_{11}-0_{01}\right)$ line often departs from the LTE ratio (Caselli et al. 1995). 
L. Pagani et al.: L183 revisited. III., Online Material p 3

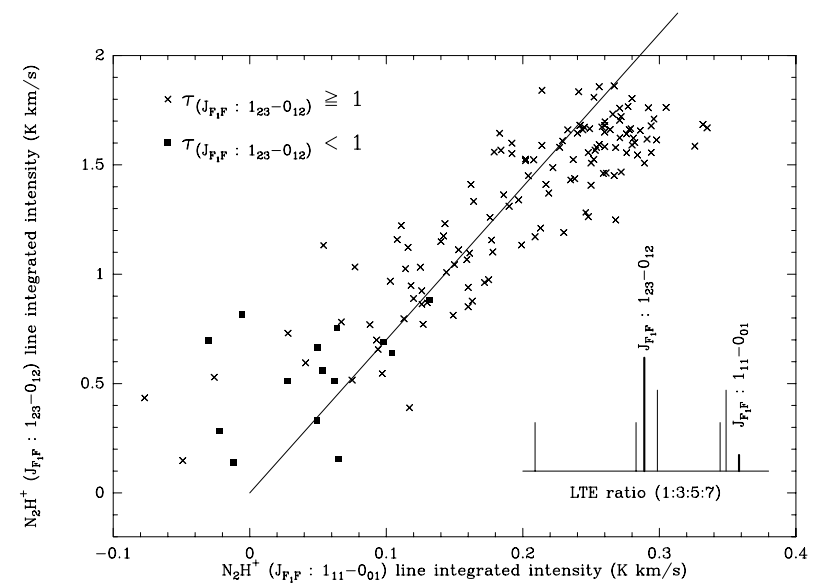

Fig. A.3. Comparison of the $\mathrm{N}_{2} \mathrm{H}^{+}\left(J_{F_{1} F}: 1_{10}-0_{01}\right)$ and $\left(J_{F_{1} F}: 1_{23}-0_{12}\right)$ line integrated intensities. Square symbols identify those spectra for which the $\left(J_{F_{1} F}: 1_{23}-0_{12}\right)$ line is optically thin. Low signal-to-noise ratios explain the presence of "optically thick" crosses in the bottom left corner. 